\title{
Evolution and Mixing of Asymmetric Holmboe Instabilities
}

\author{
by \\ Jeffrey Richard Carpenter \\ B.Sc.(Eng.), University of Guelph, 2002 \\ A THESIS SUBMITTED IN PARTIAL FULFILMENT OF \\ THE REQUIREMENTS FOR THE DEGREE OF \\ Master of Applied Science \\ in \\ The Faculty of Graduate Studies \\ (Civil Engineering) \\ The University Of British Columbia \\ September 2005 \\ (C) Jeffrey Richard Carpenter 2005
}




\section{Abstract}

When a stably stratified density interface is embedded in a region of strong velocity shear, hydrodynamic instabilities result. These instabilities lead to the development of a turbulent flow in which vertical mixing of the density field takes place. Much previous research in the field of stratified shear instability has concentrated on what has become the canonical mode in such flows - the Kelvin-Helmholtz (KH) instability. This is one of two instabilities that are present in what is termed the symmetric case, where the centres of the shear layer and density interface coincide. The other mode is the Holmboe instability, and relies on the presence of a thin density interface centred within the shear layer. In the present study the stratified shear layer is generalized to allow an offset between the centres of the shear layer and the density interface. By including this asymmetry, and keeping the density interface thin with respect to the shear layer, the asymmetric Holmboe instability is found to emerge.

The objective of the present study is to examine the evolution and mixing behavior of asymmetric Holmboe (AH) instabilities, and to compare the results to the well known $\mathrm{KH}$ and Holmboe instabilities. This is done by performing a series of direct numerical simulations (DNS). DNS has the advantage of directly resolving the smallest scales of variability present in the flow such that the turbulence and mixing characteristics do not require parameterization. In this way the mixing behavior is modeled without relying on a turbulence closure scheme. 
The simulation results show that there are two different mixing mechanisms present. The first is a feature of $\mathrm{KH}$ instabilities and is characterized by a significant overturning of the density interface. This leads to the mixing and production of intermediate density fluid causing a final density profile that is layered. The second mixing mechanism is found in $\mathrm{AH}$ and Holmboe instabilities and consists of regions of mixing and turbulence production that are located on one or both sides of the density interface. It is comprised of a cusp-like wave that periodically ejects partially mixed fluid from the top or bottom of the interface. Since the instability does not generate overturning the density interface is able to 'retain its identity' throughout the mixing event. The amount of mixing that takes place is found to be strongly dependent on the degree of asymmetry in the flow. As the asymmetry is increased the amount of mixing also increases, however, this is not necessarily an accurate representation of natural conditions as the pairing mechanism is expected to play a role in the dynamics of the flow.

The development of three-dimensional secondary structure appears to agree with previous studies (e.g.: Caulfield and Peltier (2000), Peltier and Caulfield (2003), Schowalter et al. (1994)), and consists of the formation of streamwise vortices, particularly in the gravitationally unstable regions. The presence of the density interface and the periodic ejection of interfacial fluid were also found to influence the development of these vortices. The formation and breakdown of streamwise vortices appears to be an important step in the transition to turbulence.

Since numerical models are hampered by difficulties in simulating the high Reynolds and Prandtl numbers found in nature, the geophysical relevance of the present work is also discussed in this context. 


\section{Contents}

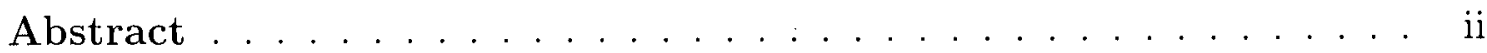

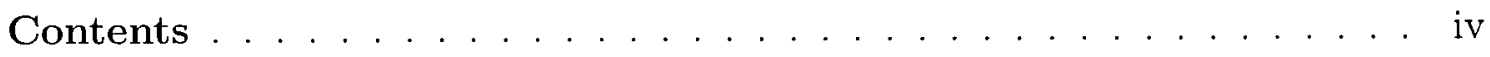

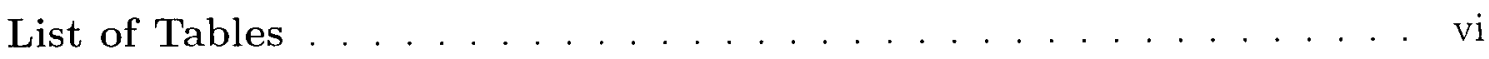

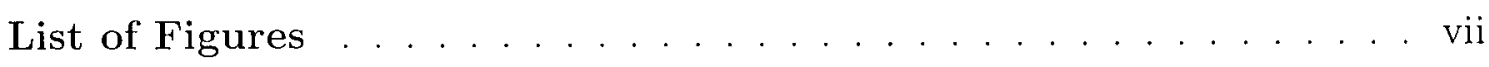

List of Symbols . . . . . . . . . . . . . . . . $\ldots$ x

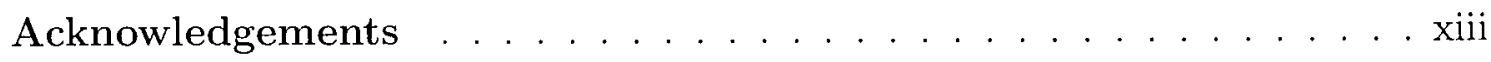

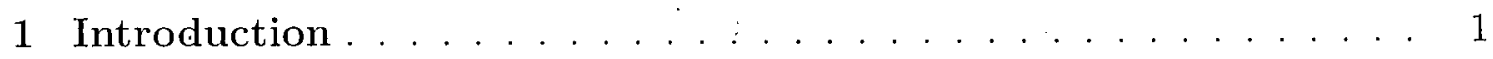

1.1 General Introduction . . . . . . . . . . . . . . . 1

1.2 Overview. . . . . . . . . . . . . . . . 3

2 Literature Review . . . . . . . . . . . . . . . . . . . 4

2.1 Theoretical Work . . . . . . . . . . . . . . 4

2.1.1 Introduction to the Taylor-Goldstein Equation and Normal Modes ... . . . . . . . . . . . . . . . . 4

2.1.2 Review of Linear Stability in Stratified Shear Flows . . . . . 6

2.2 Experimental Studies . . . . . . . . . . . . . . . . . 16

2.2.1 Laboratory Experiments and 'One-sidedness' . . . . . . . . . 16

2.2 .2 The Mixing Transition . . . . . . . . . . . . . . . . . 20

2.3 Numerical Studies. . . . . . . . . . . . . . . . . . . . . . . . 23

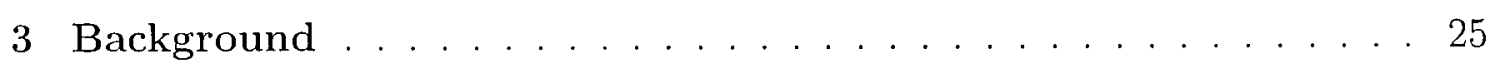

3.1 Relevant Parameters . . . . . . . . . . . . . . . . . . 28

3.2 The Partition and Transfer of Energy . . . . . . . . . . . 32

3.3 Mixing Efficiency . . . . . . . . . . . . . . . . . . . 37

4 Numerical Solution Method . . . . . . . . . . . . . . . . 39

4.1 Direct Numerical Simulations . . . . . . . . . . . . . . . . . . . . . 39

4.2 Initial and Boundary Conditions . . . . . . . . . . . . . 41 


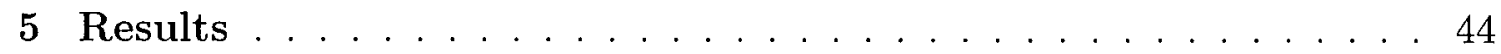

5.1 The Symmetric Case: KH and Holmboe Instabilities . . . . . . . . . 44

5.2 The Asymmetric Case: AH Instabilities and the Effects of Asymmetry 50

6 Discussion . . . . . . . . . . . . . . . . . . . . . . 64

6.1 Mixing Behavior . . . . . . . . . . . . . . . . . 64

6.2 Relevance to Geophysical Flows . . . . . . . . . . . . . 74

7 Conclusions and Future Work . . . . . . . . . . . 76

Bibliography ... . . . . . . . . . . . . . . . . . . . . 79 


\section{List of Tables}

4.1 Summary of numerical simulations performed. . . . . . . . . . . . . . 42 


\section{List of Figures}

2.1 Roll-up of a vortex sheet (from Rosenhead (1931)). . . . . . . . . 8

2.2 Stability diagram for profiles examined by Taylor (1931). Contours represent lines of constant nondimensional growth rate. . . . . . . 10

2.3 Stability diagrams showing contours of dimensionless growth rate $\alpha c_{i}$ for the symmetric case. . . . . . . . . . . . . . . . 12

2.4 Stability diagrams showing contours of dimensionless growth rate $\alpha c_{i}$ for an asymmetric case. . . . . . . . . . . . . . . . . . 14

2.5 Instabilities observed in the tilting-tube experiments of Thorpe (1968). A one-sided flow is observed in (a), while KH instabilities are shown in (b). . . . . . . . . . . . . . . . . . 17

2.6 Illustration of a mixing layer (from Breidenthal (1981)). . . . . . 18

2.7 The mixing transition in a homogenous liquid jet experiment (from Dimotakis (2000)). The change in mixing behavior is due to the mixing transition. This is achieved by the larger Re flow in (b). . . . 21

3.1 Schematic of velocity and density distribution parameters. . . . . . 29

3.2 Illustration of the relationships between the various energy reservoirs (after Winters et al. (1995)). . . . . . . . . . . . . . 35

5.1 Energy reservoirs and transfers for the $\mathrm{KH}$ simulation. The plot in (a) includes the potential energy reservoirs $P$ (solid line), $P_{B}$ (dashed line), and $\Phi$ (dotted line). In (b) both irreversible transfers $D$ (solid line), and $M$ (dashed line) are shown along with $E_{i}$ in (c). The $K_{2 d}$ (solid line) and $K_{3 d}$ (dashed line) are plotted in (d).

5.2 Plots of the density field in the $\mathrm{KH}$ simulation showing its time evolution. Slices of the $x z$-plane are taken at $y=L_{y} / 2$ with times shown in each plot.

5.3 Plots of the density field in the symmetric Holmboe simulation showing its time evolution. Slices of the $x z$-plane are taken at $y=L_{y} / 2$ with times shown in each plot. 
5.4 Energy reservoirs and transfers for the Holmboe simulation. The plot in (a) includes the potential energy reservoirs $P$ (solid line), $P_{B}$ (dashed line), and $\Phi$ (dotted line). In (b) both irreversible transfers $D$ (solid line), and $M$ (dashed line) are shown along with $E_{i}$ in (c). The $K_{2 d}$ (solid line) and $K_{3 d}$ (dashed line) are plotted in (d). . . . . 49

5.5 Energy reservoirs and transfers for the $\epsilon=0.25$ simulation. The plot in (a) includes the potential energy reservoirs $P$ (solid line), $P_{B}$ (dashed line), and $\Phi$ (dotted line). In (b) both irreversible transfers $D$ (solid line), and $M$ (dashed line) are shown along with $E_{i}$ in (c). The $K_{2 d}$ (solid line) and $K_{3 d}$ (dashed line) are plotted in (d). . . . . 51

5.6 Plots of the density field in the $\epsilon=0.25$ simulation showing its time evolution. Slices of the $x z$-plane are taken at $y=L_{y} / 2$ with times shown in each plot. . . . . . . . . . . . . . .

5.7 Cross-section in the $y z$-plane of the density field with $\vec{u}_{3 d}$ vectors overlaid for the $\epsilon=0.25$ simulation. The streamwise location is taken within the primary vortex for $x=4 h$ at $t=89$.

5.8 Energy reservoirs and transfers for the $\epsilon=0.50$ simulation. The plot in (a) includes the potential energy reservoirs $P$ (solid line), $P_{B}$ (dashed line), and $\Phi$ (dotted line). In (b) both irreversible transfers $D$ (solid line), and $M$ (dashed line) are shown along with $E_{i}$ in (c). The $K_{2 d}$ (solid line) and $K_{3 d}$ (dashed line) are plotted in (d). . . . .

5.9 Plots of the density field in the $\epsilon=0.50$ simulation showing its time evolution. Slices of the $x z$-plane are taken at $y=L_{y} / 2$ with times shown in each plot. . . . . . . . . . . . . .

5.10 Cross-section in the $y z$-plane of the density field with $\vec{u}_{3 d}$ vectors overlain for the $\epsilon=0.50$ simulation. The streamwise location is taken within the primary vortex for $x=4 h$ at $t=89 \ldots \ldots \ldots$.

5.11 Energy reservoirs and transfers for the KH simulation. The plot in (a) includes the potential energy reservoirs $P$ (solid line), $P_{B}$ (dashed line), and $\Phi$ (dotted line). In (b) both irreversible transfers $D$ (solid line), and $M$ (dashed line) are shown along with $E_{i}$ in (c). The $K_{2 d}$ (solid line) and $K_{3 d}$ (dashed line) are plotted in (d). . . . . . . .

5.12 Plots of the density field in the $\epsilon=1.0$ simulation showing its time evolution. Slices of the $x z$-plane are taken at $y=L_{y} / 2$ with times shown in each plot. . . . . . . . . . . . . .

6.1 Evolution of the $K_{3 d}$ reservoir for each simulation. . . . . . . . . 66

6.2 Comparison of the total amount of mixing in each simulation. . . . 67

6.3 Evolution of $K$ as a fraction of the initial kinetic energy $K_{0}=K(0)$ for each simulation. . . . . . . . . . . . . . . 68 
6.4 Changes in (a) the total mixing and (b) $E_{c}$ for the various asymmetries. 69 6.5 Initial and final density characteristics of the asymmetric simulations for $\epsilon=0.25$ in (a) and (b), $\epsilon=0.50$ in (c) and (d). In (a) and (c) the distribution of density elements is shown for the initial time (dark bars) and the final time (light bars). In (b) and (d) the initial (dashed line) and final (solid line) density profiles are given. All density values are given as the difference from the initial mean density. . . . . . 71

6.6 Initial and final density characteristics of the symmetric simulations. In (a) and (c) the distribution of density elements is shown for the initial time (dark bars) and the final time (light bars). The initial (dashed line) and final (solid line) density profiles are given in (b) and (d). The KH simulation corresponds to (a) and (b), whereas the Holmboe is depicted in (c) and (d). All density values are given as the difference from the initial mean density. . . . . . . . . . . 73 


\title{
List of Symbols
}

\author{
Roman Characters:
}

$c$........ Complex phase speed

$c_{r} \ldots \ldots$. Real component of the complex phase speed

$c_{i} \ldots \ldots$.... Imaginary component of the complex phase speed

$D \ldots \ldots$. Volume averaged viscous dissipation rate

$D_{p} \ldots \ldots$. Rate of energy gain due yo diffusion of the initial density profile

$d \ldots \ldots \ldots$. Dimensional asymmetry offset

$E_{c} \ldots \ldots$. Cumulative mixing efficiency

$E_{i} \ldots \ldots \ldots$ Instantaneous mixing efficiency

$g$........ Gravitational acceleration

$g^{\prime} \ldots \ldots \ldots$ Reduced gravitational acceleration

$H \ldots \ldots$. Buoyancy flux

$h \ldots \ldots \ldots$. Shear layer thickness

$i \ldots \ldots$... Imaginary unit, $\sqrt{ }-1$

$J$........ Bulk Richardson number

$K \ldots \ldots$. Volume averaged kinetic energy

$K_{0} \ldots \ldots \ldots$. Initial kinetic energy

$\bar{K} \ldots \ldots \ldots$ Kinetic energy of the mean flow

$K_{2 d} \ldots \ldots$. Two-dimensional kinetic energy

$K_{3 d} \ldots \ldots$. Three-dimensional kinetic energy

$k \ldots \ldots \ldots$. Dimensional wavenumber

$\hat{k}$........ Unit vector in the vertical (z) direction

$L_{x} \ldots \ldots$. Domain length in the streamwise direction

$L_{y} \ldots \ldots \ldots$. Domain length in the spanwise direction

$L_{z} \ldots \ldots$... Domain length in the vertical direction

$L_{z}^{*} \ldots \ldots \ldots$ Dimensionless vertical domain length

$M$........ Rate of mixing

$N_{x} \ldots \ldots \ldots$ Number of grid points in streamwise direction (course grid)

$N_{y} \ldots \ldots \ldots$ Number of grid points in spanwise direction (course grid)

$N_{z} \ldots \ldots \ldots$ Number of grid points in vertical direction (course grid)

$P \ldots \ldots$ Total potential energy

$P_{A} \quad \ldots \ldots$. Available potential energy 


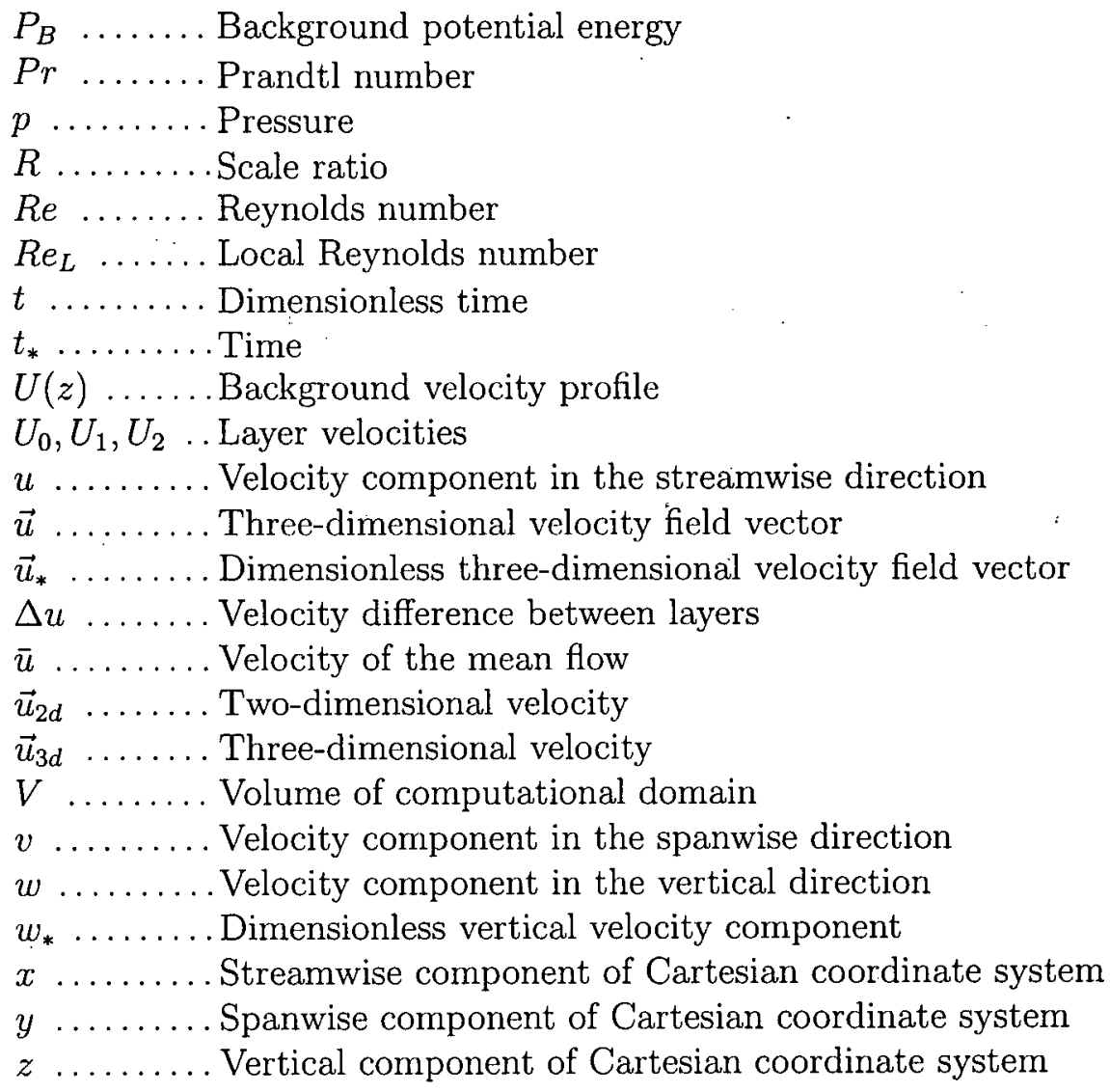

Greek Characters:

$\alpha \ldots \ldots \ldots$ Dimensionless wavenumber

$\epsilon \ldots \ldots$ Dimensionless asymmetry factor

$\varepsilon \ldots \ldots$ Local viscous dissipation rate

$\eta \ldots \ldots$. Density interface thickness

$\kappa \ldots \ldots \ldots$ Diffusion coefficient of density

$\lambda \ldots \ldots$..... Wavelength

$\nu \ldots . .$. . Coefficient of kinematic viscosity

$\rho \ldots \ldots . .$. Density

$\rho_{*} \ldots \ldots$ Dimensionless density

$\rho_{0} \ldots \ldots \ldots$ Reference density

$\Delta \rho \ldots \ldots$ Density difference between layers

$\rho_{1}, \rho_{2}, \rho_{3} \ldots$ Layer densities

$\bar{\rho}(z) \ldots \ldots$ Initial density profile 
$\rho_{B}(z) \ldots \ldots$ Background density profile

$\bar{\rho}_{\text {top }} \ldots \ldots$... Average density on top boundary of domain

$\bar{\rho}_{\text {bottom }}$..... Average density on bottom boundary of domain

$\sigma \ldots \ldots$. Dimensionless growth rate

$\tau$........ Arbitrary time scale

List of Abbreviations:

AH .......Asymmetric Holmboe

$D N S \ldots$. . Direct numerical simulation

$K H \ldots \ldots$ Kelvin-Helmholtz

$S W 03 \ldots$... Smyth and Winters (2003)

$T G \ldots . .$. Taylor-Goldstein 


\section{Acknowledgements}

This thesis would not be anywhere near the product it is without the help of many people. I'd first like to acknowledge not only the guidance and insight provided by my supervisor Greg Lawrence but also his generosity in providing the freedom for me to pursue any and all opportunities as they arose. The help of Bill Smyth in both getting the model working and providing input on the results has been invaluable. It is my hope that this correspondence will continue and grow in the future. I have also benefited from the programming prowess and determination of Sheng $\mathrm{Li}$ in the dreaded debugging stages of this project. I've had many entertaining and helpful discussions with Ted Tedford on topics closely related to this thesis. Finally, thank you to my family and friends. 


\section{Chapter 1}

\section{Introduction}

\subsection{General Introduction}

This thesis studies the process of turbulence generation and mixing of a stably stratified density interface. There are many different mechanisms by which turbulent mixing is accomplished, and a focus here is placed on the case in which mixing results from the presence of a velocity shear. This shear allows hydrodynamic instabilities to form on the density interface which lead the flow to a turbulent state whereby irreversible mixing of the density profile is accomplished. The character of this transition process as well as the resulting turbulence is highly dependent on the type of instability that forms. This is in turn dependent on both the velocity and density distributions.

Since density stratification is virtually ubiquitous in the natural environment the suppression of turbulence in regions of stable stratification plays an important role in the physics of many geophysical systems. It reduces vertical transport rates to near-molecular levels and serves to isolate different fluid layers from one another. When enough energy can be supplied by a velocity shear to generate instabilities, the result is enhanced rates of vertical transport (i.e., a mixing of the density interface). This scenario has major environmental implications ranging from the dispersion of atmospheric pollutants, to the supply of nutrients to the photic zone in lakes and 
oceans.

There are a number of documented processes by 'which instability results from the shearing of a stable stratified layer. In oceanographic and limnological scenarios the mixing is often forced to occur across relatively sharp density gradients. The formation of sharp density interfaces can be due to a number of different fluid mechanical processes including shear instability itself (see Turner (1973); Peltier and Caulfield (2003)). The maintenance of these sharp gradients is due primarily to their high levels of static stability and the high Prandtl number environments they are found in (i.e., temperature and salt stratified water bodies).

The stability characteristics of sharp density gradient shear layers permit a departure from the canonical Kelvin-Helmholtz $(\mathrm{KH})$ mode of instability and allows for the formation of Holmboe modes. The mixing behavior of Holmboe modes is a relatively unexplored area of research with the only direct quantitative study being that of Smyth and Winters (2003) (SW03). This work focused on the symmetric Holmboe instability (simply referred to herein as the Holmboe instability), a case that results when the centre of the shear layer and the centre of the density interface coincide. SW03 found that for the single point in parameter space studied, the Holmboe instability was able to accomplish a greater degree of mixing than the $\mathrm{KH}$ instability. This highlights the possible importance of Holmboe modes in contributing to the mixing of sharp density interfaces.

In this study the Holmboe mode is generalized to the asymmetric case, where an offset exists between the centre of the shear layer and the density interface. The resulting instabilities are referred to as asymmetric Holmboe $(\mathrm{AH})$ instabilities. The mixing behavior of $\mathrm{AH}$ instabilities has not been studied previously. In 
fact, the $\mathrm{AH}$ instability has thus far existed primarily in theoretical circles, with only a handful of observations in laboratory experiments. Therefore, the following chapters are devoted to studying the evolution and mixing behavior of AH instabilities, and comparing the findings to previous laboratory experiments and theoretical predictions.

\subsection{Overview}

This study constitutes a first step at evaluating the importance of asymmetry in the development of instabilities and mixing in stratified shear flows. In the course of this study, which includes a comparison between $\mathrm{KH}$, Holmboe, and asymmetric Holmboe instabilities, I have relied on the direction set out in SW03. In SW03 a comparison is made between the $\mathrm{KH}$ and Holmboe instabilities in terms of mixing and turbulence generation in the same region of parameter space. As the present study can be viewed as an extension of the results found in SW03, it is important to give proper acknowledgment.

Interpretation of the simulation results was done in collaboration with my supervisor Greg Lawrence, and this work should not be viewed solely as the work of myself as an exclusive author. 


\section{Chapter 2}

\section{Literature Review}

\subsection{Theoretical Work}

\subsubsection{Introduction to the Taylor-Goldstein Equation and Normal Modes}

Much early work on density stratified shear instabilities focused on the theoretical prediction of unstable flow configurations. This led to the development of what is now known as the Taylor-Goldstein (TG) equation. Based on the pioneering work of Taylor (1931) and Goldstein (1931), the TG equation describes the fate of linear twodimensional, monochromatic, sinusoidal disturbances in a density stratified fluid. The principle behind the TG equation is that if an infinitesimally small disturbance applied to given basic velocity and density configurations is found to grow in time, then the flow is deemed unstable, and results in the growth of an instability. This instability does not necessarily lead to turbulence, merely a change in the basic flow pattern. This description will become more clear in the following paragraphs where the method is described in more detail.

To determine the stability of a given flow, the variables of interest are decomposed into mean and fluctuating components, where the mean components become representative of the basic flow. Once the decompositions are substituted into the 
equations of motion, the resulting equations are linearized by neglecting the products of the perturbation terms. Just as neglecting the higher order terms in a Taylor's series expansion, the resulting solution is only valid for small deviations from the basic state. This requires that the perturbation quantities do not become too large. Linear theory is thus only usually assumed to apply to the evolution of infinitesimal disturbances. Since - even after linearizing - the solutions of the resulting equations are often extremely difficult to solve analytically, a number of simplifying assumptions are made. These most often include solving for the twodimensional flow of an incompressible, inviscid fluid with negligible diffusion of the scalar field. It is also convenient to make the Boussinesq approximation, which states that differences in density can be neglected in all inertial terms, but included in the buoyancy term. Justification for the use of the approximation in this study can be seen by the small density differences that are typically observed in flows of practical importance, particularly $\Delta \rho / \rho=O\left(10^{-3}\right)$ in typical ocean environments (Turner, 1973).

Once the approximations and procedures outlined above have been made the result is a single linear homogeneous partial differential equation describing the evolution of small perturbations from the basic flow. The most common method of obtaining solutions for this equation is by the method of normal modes. This method searches for wavelike solutions in $x$ and $t$ whose amplitude varies as a function of $z$. Specifically, solutions are assumed to be of the form

$$
\phi(x, z, t)=\hat{\phi}(z) e^{i k(x-c t)}
$$

where $k$ is the wavenumber, $c$ is the phase speed of the wave, and $\phi$ represents the 
perturbation quantity of interest (e.g.: vertical velocity, density). This leads to the TG equation

$$
\hat{\phi}^{\prime \prime}+\left[\frac{N^{2}}{(U-c)^{2}}-\frac{U^{\prime \prime}}{(U-c)}-k^{2}\right] \hat{\phi}=0
$$

where the primes denote differentiation with respect to $z$, and the basic flow is represented by the velocity profile $\vec{u}=(U(z), 0)$ and the density profile $\rho=\bar{\rho}(z)$. The buoyancy frequency is represented by $N(z)=\left(g \rho^{\prime} / \rho_{0}\right)^{1 / 2}$. The solutions to (2.2) constitute an eigen-problem involving the unknown eigenfunction, $\hat{\phi}(z)$, and eigenvalues, $c=c_{r}+i c_{i}$, which may be complex. Each solution pair $\{\hat{\phi}, c\}$ is referred to as a normal mode (or simply just a mode), and correspond to specific values of the wavenumber and bulk Richardson number, $J=g^{\prime} h /(\Delta u)^{2}$, where $g^{\prime}=\left(\Delta \rho / \rho_{0}\right) g$ is the reduced gravitational constant. Here $\Delta \rho, \Delta u$ are representative density and velocity scales respectively, $h$ is a length scale, and $\rho_{0}$ is a reference density. The basic flow is deemed to be unstable when the eigenvalue has a positive imaginary component, i.e., $c_{i}>0$. This can be seen by substituting into (2.1) and noting that the solution is proportional to a factor growing exponentially in time (i.e., $\phi \propto e^{k c_{i} t}$ ). For this reason, the growth rate of the mode is defined by $k c_{i}$ and the phase speed is given by $c_{r}$.

\subsubsection{Review of Linear Stability in Stratified Shear Flows}

In general, solutions to the Taylor-Goldstein equation (2.2) must be obtained by complicated methods involving numerical schemes for all but the simplest cases. The difficulty arises from the fact that the coefficients are not constant. However, if piecewise-linear profiles are chosen to represent velocity and density then two of 
the coefficients vanish and (2.2) simplifies to

$$
\hat{\phi}^{\prime \prime}-k^{2} \hat{\phi}=0
$$

which allows for analytical solutions in most cases. Due to this simplification, a great majority of work in developing solutions has been performed on profiles that are piecewise-linear (see for example Howard and Maslowe (1973); Lawrence et al. (1991); Haigh and Lawrence (1999)).

The simplest scenario of shear flow instability that can be analysed using linear theory is the homogenous case where the velocity profile is represented by

$$
U(z)=\left\{\begin{aligned}
U_{0} & , \text { if } z>0 \\
-U_{0}, & \text { if } z<0
\end{aligned}\right.
$$

By applying the boundary condition that the velocity must vanish as $z \rightarrow \pm \infty$, and the matching condition that the pressure is continuous at the discontinuity, a dispersion relation is obtained for the complex phase speed (see Drazin and Reid $(1982, \S 28)$ for details). The results, which were first developed by Helmholtz (1868), show that the growth rate is directly proportional to the wavenumber, and the phase speed stationary with respect to the mean flow. This corresponds to the instability of a vortex sheet, and is characterized in the early stages of nonlinear growth by a rolling-up of the sheet into a periodic train of billows, as shown in figure 2.1 below. This is a special case of the density stratified flow studied by Kelvin (1871), which consists of two layers of velocity $U_{1}$ and $U_{2}$, each with density $\rho_{1}$ and $\rho_{2}$, respectively. In this case the results of linear stability predict that the flow is unstable for a range 


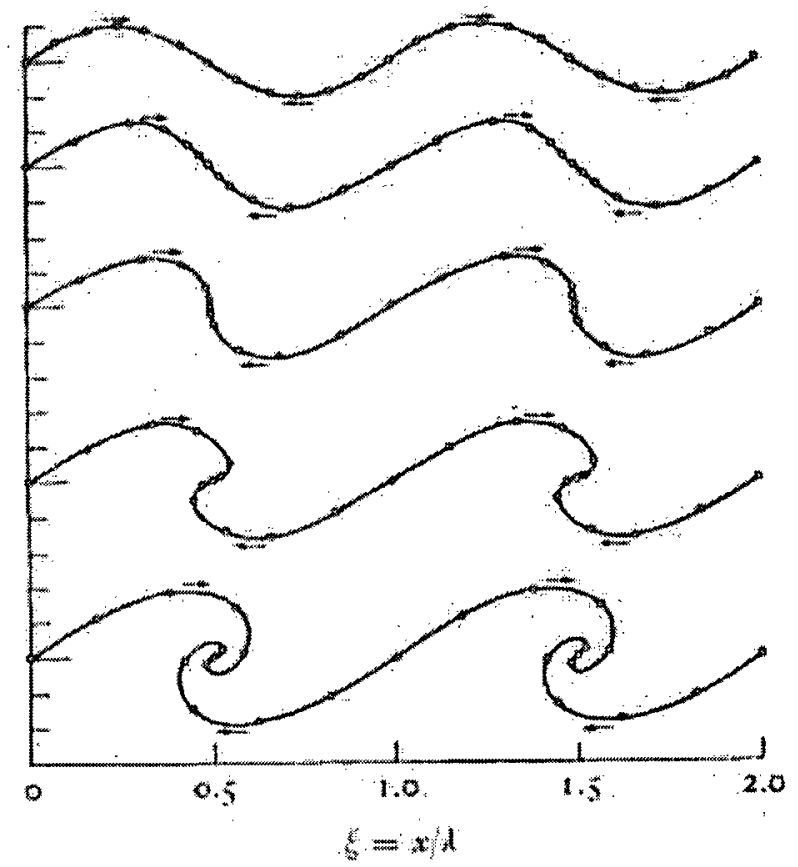

Figure 2.1: Roll-up of a vortex sheet (from Rosenhead (1931)). 
of wavenumbers satisfying the relation $k \rho_{1} \rho_{2}\left(U_{1}-U_{2}\right)^{2}>g\left(\rho_{1}^{2}-\rho_{2}^{2}\right)$. This shows that the presence of stable stratification is responsible for stabilizing the flow for a certain range of wavenumbers.

The next major developments came from Taylor (1931) and Goldstein (1931) in their analysis of piecewise-linear profiles that incorporated shear layers of finite thickness over layers of stable density stratification. The main result of these works was the development of the Taylor-Goldstein equation (2.2), but they also revealed part of the subtle relationship that density stratification can play in determining the stability of a flow. Stable stratification is generally considered to exhert a stabilizing influence on a given flow. This can be seen in the example of the vortex sheet just disscussed, where the addition of stable density stratification stabilized the flow for longer wavelengths, namely $\lambda>\pi(\Delta U)^{2} / g^{\prime}$. It can also be thought of intuitively by noting that it takes additional potential energy in moving a fluid parcel across the density interface. Taylor (1931) showed that to think of stratification solely as a stabilizing influence was an oversimplification; that it is possible for stable stratification to render a previously stable flow unstable. This can be seen by examining the following profiles

$$
U(z)=z, \quad \bar{\rho}(z)= \begin{cases}\rho_{1} & \text { for } z>d \\ \rho_{2} & \text { for } d<z<-d \\ \rho_{3} & \text { for } z<-d\end{cases}
$$

where $\rho_{2}=\rho_{1}+\Delta \rho$ and $\rho_{3}=\rho_{1}+2 \Delta \rho$. The basic flow defined above corresponds to plane Couette flow with three homogenous layers of different density superimposed. Performing a linear stability analysis on these profiles yields the stability diagram 


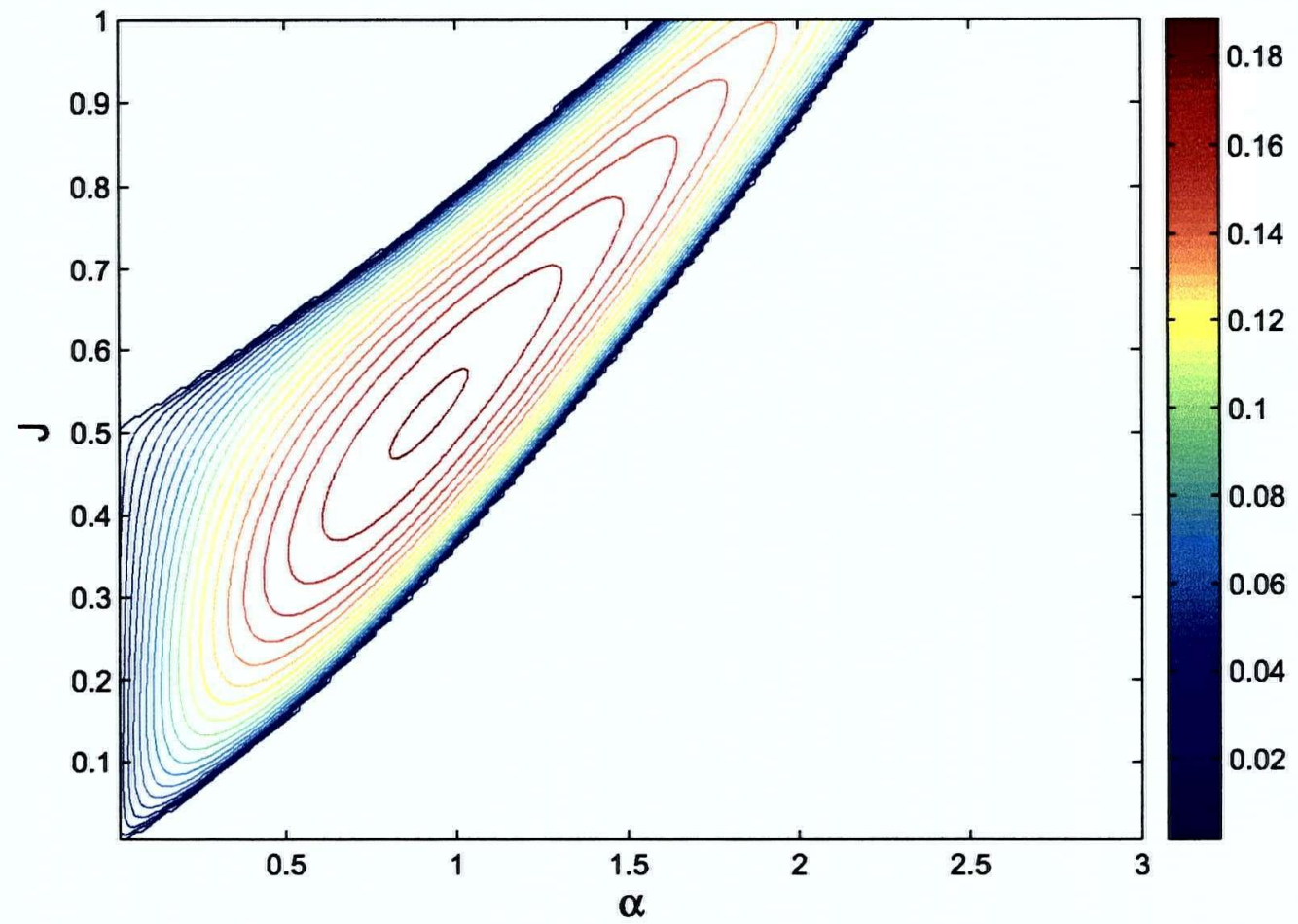

Figure 2.2: Stability diagram for profiles examined by Taylor (1931). Contours represent lines of constant nondimensional growth rate.

in figure 2.2. From the stability diagram it can be seen that the flow is stable for $J=0$, corresponding to the homogenous case. It should be noted however, that Couette flow is only stable in the inviscid limit - meaning that for large enough (but finite) values of the Reynolds number, viscosity will destabilize the flow (see Drazin and Reid (1982)). In this respect, Taylor found that the flow was destabilized by the addition of stratification. It can also be seen in figure 2.2 that the maximum growth rate is attained at a value of $J \approx 0.52$. This indicates that the mechanism for instability relies on a certain amount of stratification to grow. Howard and Maslowe 
(1973) give the following explanation for the mechanism:

The motion consists approximately of two internal waves on the two density interfaces, the upper one propagating to the left relative to the local flow speed at such a velocity as to stand still relative to the origin, and the lower one similarly standing still relative to the origin. The pressure fields of each wave perturb the other a little, and in such a phase that they amplify each other.

Holmboe (1962) developed further the ideas of both stratification as exerting a possibly destabilizing influence, and the interaction of waves as a mechanism of linear growth. Using a method similar to normal modes, Holmboe examined a case where the density interface is much smaller than, and centred within, a larger shear layer. He represented this flow with the following profiles:

$$
U(z)=\left\{\begin{array}{cl}
U_{0} & \text { for } z>d \\
\frac{U_{0}}{d} z & \text { for } d<z<-d \\
U_{0} & \text { for } z<-d
\end{array} \quad \bar{\rho}(z)=\left\{\begin{array}{cl}
\rho_{1} & \text { for } z>0 \\
\rho_{2} & \text { for } z<0
\end{array}\right.\right.
$$

The results of Holmboe's analysis showed that the flow was unstable to two different types of instabilities: (i) the Kelvin-Helmholtz instability, characterized by a stationary phase speed with respect to the mean flow also present in the unstratified case, and (ii) Holmboe's instability, an oscillatory instability with nonzero phase speed that is the only instability to occur for $J>0.07$. These instabilities occupy two different regions of the $J, \alpha$-plane as seen in the stability diagram of figure 2.3. Holmboe's instability was predicted to consist of two simultaneously unstable modes that had equal growth rates and equal but opposite phase speeds. 


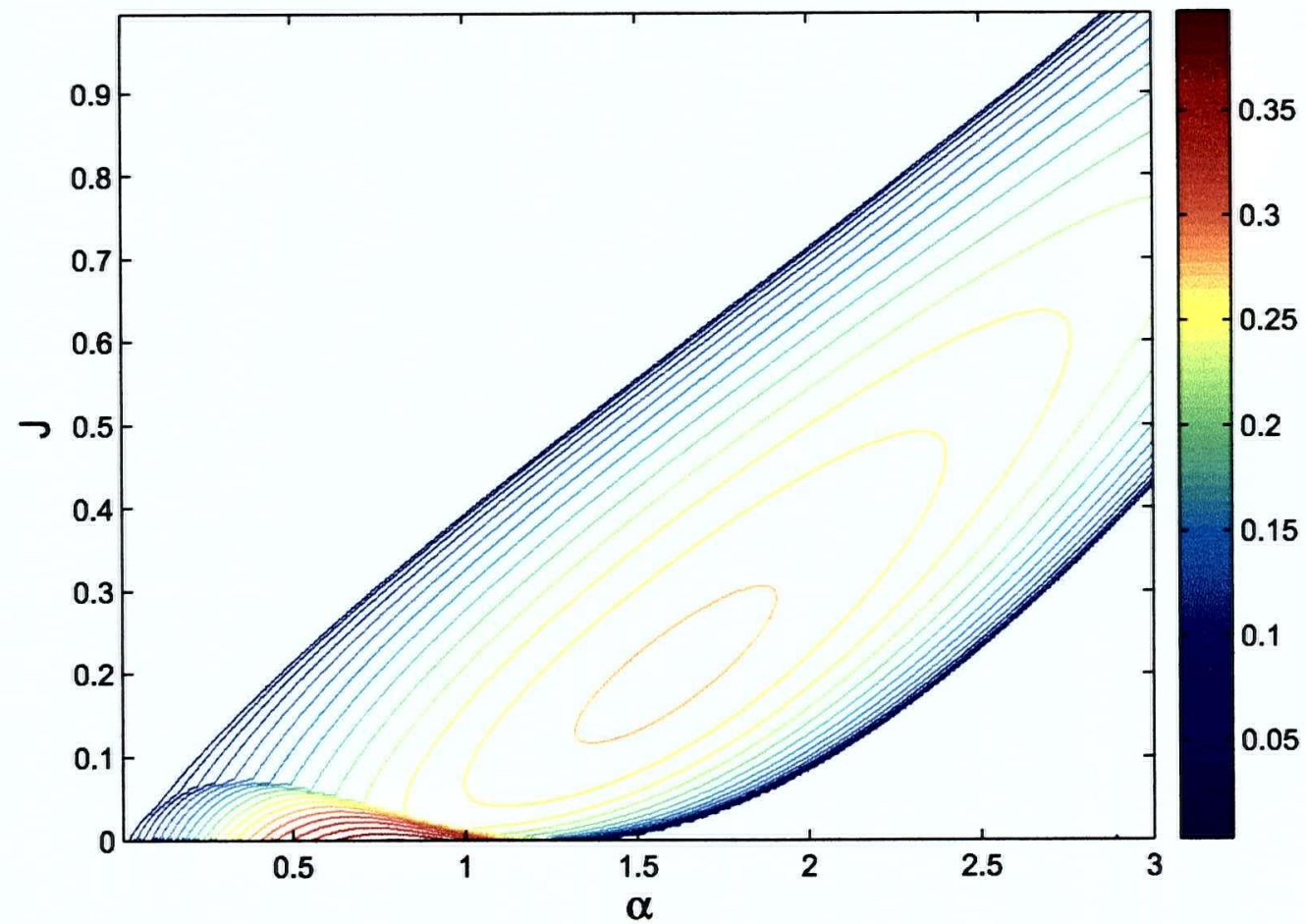

Figure 2.3: Stability diagrams showing contours of dimensionless growth rate $\alpha c_{i}$ for the symmetric case. 
The mechanism responsible for the linear growth of Holmboe's instability was first discussed by Holmboe (1962) and further elaborated upon by Baines and Mitsudera (1994). Similar to the Taylor case examined above, it consists of the interaction of disturbances on the vorticity interfaces (the locations where the vorticity of the shear layer, $d U / d z$, changes abruptly) with internal gravity waves on the density interface. In this respect, two Holmboe modes are formed that travel at speeds comparable to internal gravity waves, and are centred above and below the density interface.

Motivated by experimental results (cf. §2.2.1), Lawrence et al. (1991) modified Holmboe's original model (2.6) by including a density interface that is offset from the centre of the shear layer. The profiles now take the form

$$
U(z)=\left\{\begin{array}{rl}
U_{0} & \text { for } z>d \\
\frac{U_{0}}{d} z & \text { for } d<z<-d \\
-U_{0} & \text { for } z<-d
\end{array} \quad \bar{\rho}(z)=\left\{\begin{array}{cl}
\rho_{1} & \text { for } z>\delta \\
\rho_{2} & \text { for } z<\delta
\end{array}\right.\right.
$$

The stability diagram for one such asymmetric case is shown in figure 2.4. From this it can be seen that by including this offset the growth rate of one of the two modes of instability is preferentially destabilized with respect to the other. The stronger mode being that on the upper side of the density interface where a greater amount of shear layer vorticity resides, and the weaker mode occupying the lower vorticity side of the interface. Other interesting features that result include the vanishing of the region of zero phase speed that is associated with the $\mathrm{KH}$ instability, as well as the different wavenumbers of maximum growth rate for each of the two modes. The splitting of the regions of instability into two separate modes with a nonzero 


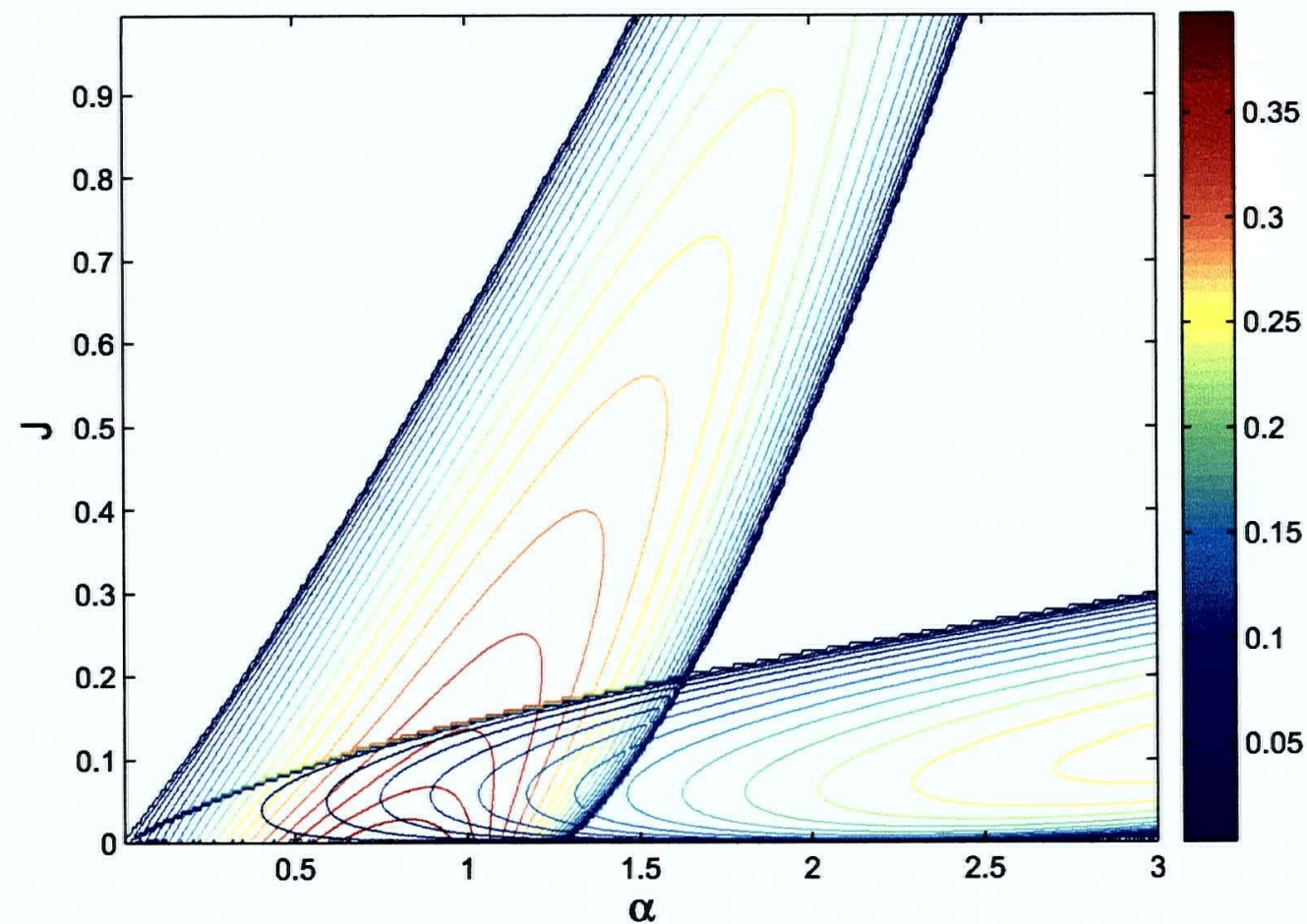

Figure 2.4: Stability diagrams showing contours of dimensionless growth rate $\alpha c_{i}$ for an asymmetric case. 
phase speed indicates that, according to the framework of Baines and Mitsudera (1994), the resulting modes of instability are Holmboe-like modes resulting from the interaction of vorticity disturbances and internal waves.

The linear stability results of Lawrence et al. (1991) was extended by Haigh (1995) to include both an offset in the density interface and asymmetrically placed horizontal (free-slip) boundaries. Linear stability analysis was performed on piecewiselinear and hyperbolic tangent velocity and density profiles that included the effects of scalar diffusion and viscosity. The hyperbolic tangent profiles being found to more accurately characterize the distributions found in natural settings (for example in the saltwedge flows of Yonemitsu et al. (1996)), and to be a close approximation to the error function profiles that result from molecular diffusion. Linear stability analysis of smooth profiles accounting for viscosity had been accomplished earlier by Maslowe and Thompson (1971) where it was found that viscosity is only effective in damping weakly amplified waves near the stability boundaries. This agrees with the results of Haigh (1995) who found that decreasing the Reynolds number led to a shift to lower wavenumbers of the most amplified mode, as well as a narrowing of the stability boundaries. However, the Richardson number is the dominant parameter governing the stability of unbounded shear flows (Maslowe and Thompson, 1971). The introduction of continuous hyperbolic tangent profiles did not greatly change the results found in the piecewise-linear case of Lawrence et al. (1991) from (2.7). 


\subsection{Experimental Studies}

\subsubsection{Laboratory Experiments and 'One-sidedness'}

Attempts to study Holmboe's instability and the theoretical predictions of Holmboe (1962) in the laboratory were often found to result in a 'one-sidedness'. This term is now used in the literature to describe flows in which the disturbances and mixing generated as a result of instability appear in only one of the two layers (see Maxworthy and Browand (1975); Lawrence et al. (1991); Yonemitsu et al. (1996)). It has been identified as a common feature of mixing layer facilities, and is due to the asymmetry introduced between the centre of the shear layer and the centre of the density interface (Lawrence et al. $(1991,1998)$ ).

The first laboratory experiments that appeared to confirm the existence of instabilities other than KH in a stratified shear flow were those of Thorpe (1968). Using a tilting tube, Thorpe was able to create a nearly parallel flow for a short period of time until surges formed at the channel ends reached the centre of the tube. Some of the best known photographs of $\mathrm{KH}$ billows were taken from these experiments. These are shown in figure $2.5 \mathrm{~b}$ along with some of the observed one-sided instabilities (figure 2.5a), where the disturbances and mixing were confined to the upper side of the density interface. These one-sided instabilities appeared as cusp-like waves that travelled with respect to the mean flow. Since Holmboe instabilities are predicted to comprise of both left and right propagating modes, these instabilities do not appear to be of the type Holmboe predicted (though they resemble his predictions in many other respects). In this study Thorpe (1968) notes that throughout the evolution and mixing in these one-sided experiments the density interface 'retains its identity'. 
(a)

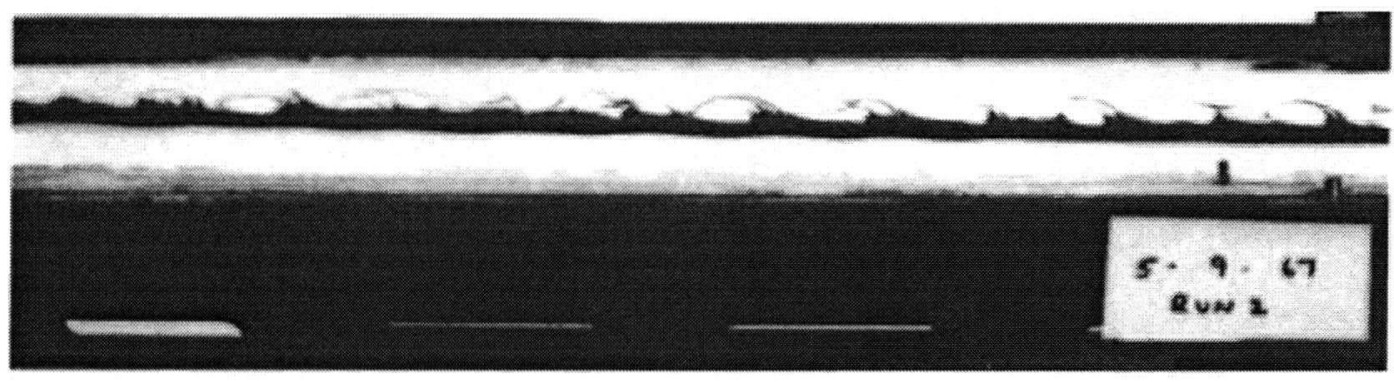

(b)

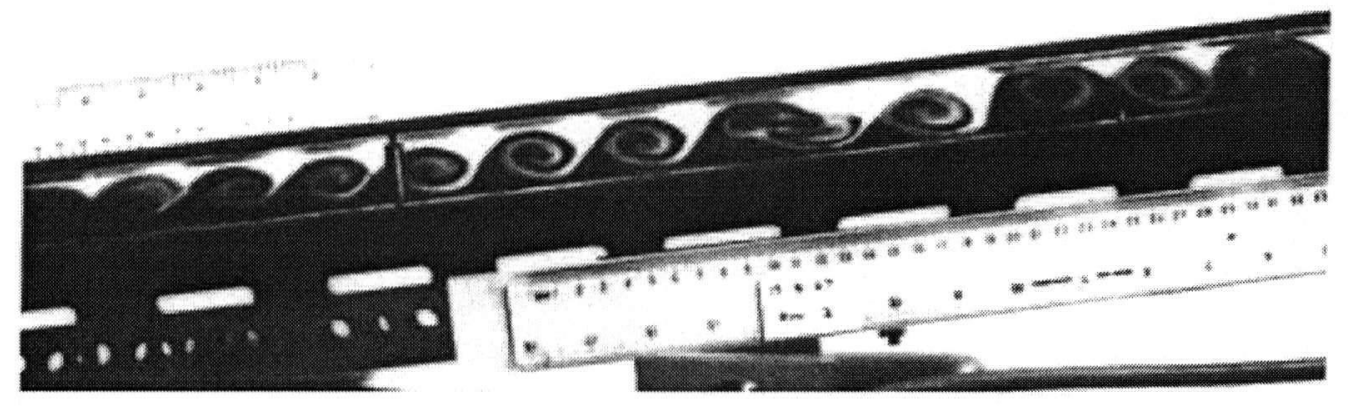

Figure 2.5: Instabilities observed in the tilting-tube experiments of Thorpe (1968). A one-sided flow is observed in (a), while $\mathrm{KH}$ instabilities are shown in (b). 
In this respect the mixing is fundamentally different than that present in $\mathrm{KH}$ instabilities, where it is accomplished by a complete overturning and subsequent mixing of the interface.

The only laboratory investigation that has been carried out with the intent of studying asymmetric stratified shear layers is that of Lawrence et al. (1991). The experiments were performed in mixing layer facilities, and consist of two streams of fluid with densities $\rho_{1}$ and $\rho_{2}$, and velocities $U_{1}$ and $U_{2}$ separated by a splitter plate as shown in figure 2.6. By adjusting the velocity difference between the two

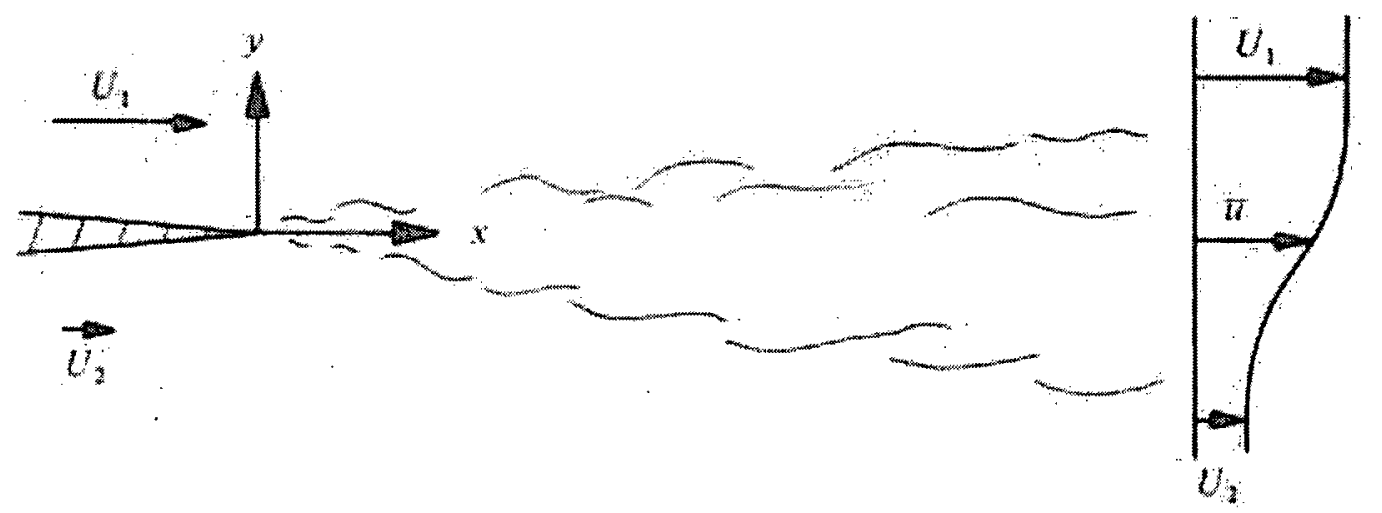

Figure 2.6: Illustration of a mixing layer (from Breidenthal (1981)).

streams, the stability characteristics of the flow can be examined. Asymmetry results in mixing layer experiments due to the formation of differing boundary layers on the high- and low-speed side of the splitter plate leading to a greater amount of vorticity in the high-speed layer (as described in Lawrence et al. (1998)). The asymmetry of the shear layer in the experiments of Lawrence et al. (1991) was found to produce instabilities with 'one-sided' behavior, and were identified as asymmetric instabilities. 
Since the lower layer was salt stratified, the thickness of the density interface is expected to be much less than the vorticity layer, and Holmboe-like instabilities are expected for sufficiently high levels of stratification. This appears to be the case as the instabilities took the form of cusp-like waves which occasionally ejected dense fluid into the dominant layer. The mixing characteristics of the resulting instabilities were not quantified however.

Similar experiments were conducted by Schowalter et al. (1994) which focused on the formation of secondary instabilities in homogenous and stratified mixing layers. In this study a mixing layer facility was used with a serrated splitter plate edge to inject spanwise perturbations, while the primary streamwise instability was mechanically forced by moving the splitter plate at a certain frequency. The experiments were also noted to have a degree of asymmetry associated with them, though the amount of asymmetry is not quantified. The secondary instabilities took the form of streamwise vortices, as have been observed in a number of other studies (e.g.: Breidenthal (1981); Bernal and Roshko (1986); Caulfield and Peltier (2000)). In the more heavily stratified experiments, streamwise vortices were enhanced by the unstable stratification in the overturned regions of the resulting billows. These streamwise vortices are expected to lead the flow to a turbulent transition. This has been confirmed in the case of stratified $\mathrm{KH}$ instabilities in the numerical simulations of Caulfield and Peltier (2000) where the transition to turbulence led to a rapid increase in the rate of mixing of the scalar field. 


\subsubsection{The Mixing Transition}

The role that secondary structures and the onset of small-scale three-dimensional motions have on the mixing of fluid in homogenous shear layers was studied experimentally by Breidenthal (1981). Here it was found that the amount of mixing between the two streams increased by an order of magnitude once small-scale threedimensional motion set in. This phenomenon has been termed the 'mixing transition', and is found to be a function of the local Reynolds number, $\operatorname{Re}_{L}$ (Dimotakis, 2000). In fact, Dimotakis (2000) predicts that the mixing transition occurs in all flows for which an appropriately defined $R e_{L} \gtrsim 1-2 \times 10^{4}$. The significance of this result, Dimotakis (2000) states, is that $R e_{L}$ becomes large enough such that a fully-developed turbulent flow is achieved. It is further conjectured that "a necessary condition for fully-developed turbulence ... is the existence of a range of scales that are uncoupled from the large scales, on the one hand, and free from the effects of viscosity, on the other" (Dimotakis, 2000). Therefore, the mixing transition is not the result of small-scale three-dimensional motions alone, but the generation of turbulence at a sufficiently high $R e_{L}$ so that it may become fully-developed. This transition denotes a significant change in the dependence of the mixing rate on $R e$ and $\operatorname{Pr}$, where $\operatorname{Pr}=\nu / \kappa$ is the ratio of the two diffusivities of momentum (or vorticity), $\nu$, and that of the scalar quantity, $\kappa$. The study of Breidenthal (1981) concluded that the effect of $\operatorname{Pr}$ on the mixing at low $R e$ is pronounced, while at high Re the mixing changes by only a factor of 2 or less for a three orders of magnitude variation in $\operatorname{Pr}$. Stated another way, above the mixing transition the generation of mixed fluid is essentially independent of $\mathrm{Pr}$.

There are numerous examples of flows for which this mixing transition exists, 
and can be recognized by a qualitative change in the composition of the scalar field. This is illustrated in figure 2.7 for the case of a homogenous liquid jet. Here figure

(a)

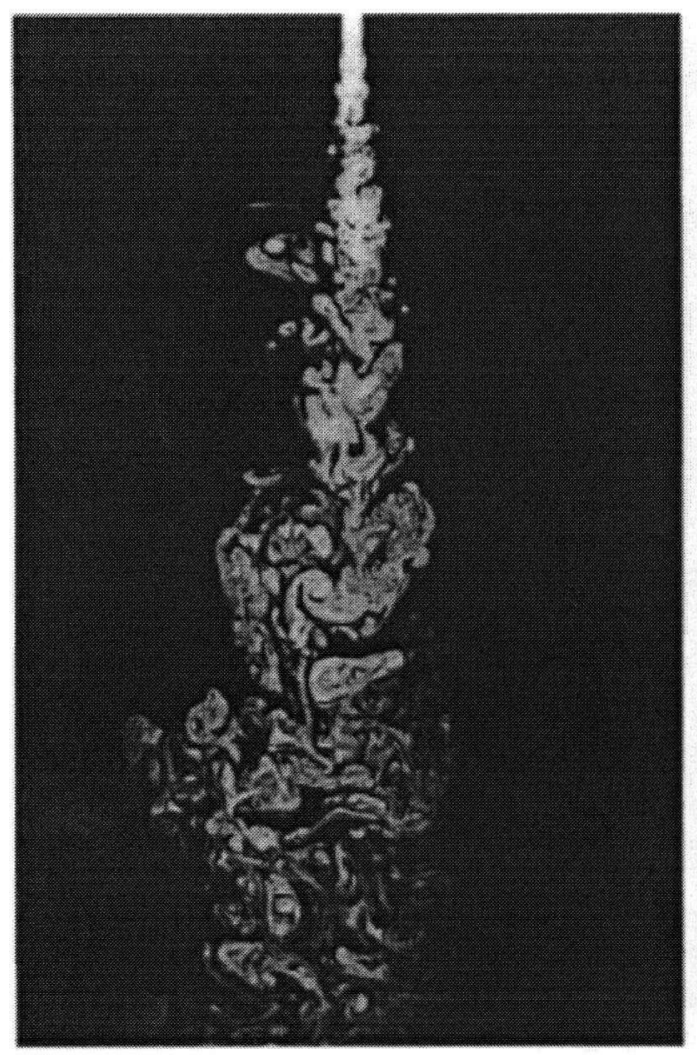

(b)

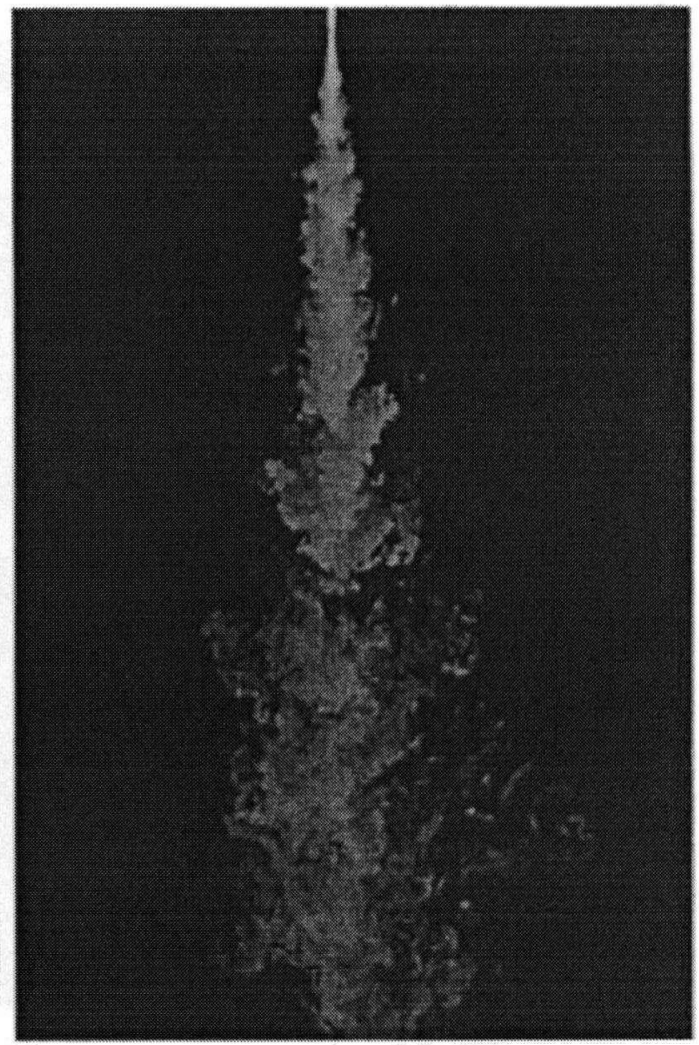

Figure 2.7: The mixing transition in a homogenous liquid jet experiment (from Dimotakis (2000)). The change in mixing behavior is due to the mixing transition. This is achieved by the larger Re flow in (b).

2.7a corresponds to the scalar field for a flow that has developed instabilities and turbulence, but has not undergone the mixing transition. The effect of the mixing transition on the scalar field for a similar experiment at a larger $R e$ is shown in figure $2.7 \mathrm{~b}$, where large amounts of mixed fluid and the presence of small-scale motion can be seen. 
Although the mixing transition has been well documented in homogenous flows, it appears that little work has been undertaken to study this phenomenon in stratified shear layers. A qualitative change in the mixing behavior of the scalar field was noted in the experiments of Pawlak and Armi (1998). These consisted of a spatially-accelerating stratified shear generated by the down-slope flow of a dense layer over a topographic sill. Entrainment between the two layers occurs through the $\mathrm{KH}$ instability while mixing is observed downstream, after the formation of turbulence on the interface. At the final downstream position of the developing mixing layer a $R e_{L} \approx 5 \times 10^{3}$ is attained, and the beginnings of the mixing transition are observed.

Evidence of a mixing transition is present in the numerical simulations of $\mathrm{KH}$ instabilities in Caulfield and Peltier (2000). These results showed heightened levels of mixing that accompanied the onset of small-scale turbulent motions after the breakdown of both the primary billow and the secondary streamwise vortices. This behavior is thought to be linked to the mixing transition as the flow is shown to be close to the critical Reynolds number criterion with $R_{L} \sim 10^{4}$ (see Peltier and Caulfield (2003)). However, the sudden jump in mixing associated with the transition can be seen to decrease in strength as the stratification is increased. It is possible that the growth of $R e_{L}$ with the shear layer thickness is sufficiently suppressed by the stratification such that the critical mixing transition criterion is not reached. In this sense, the mixing transition may not play as strong a role in the dynamics of naturally developing shear instabilities in strongly stratified fluids as it does with homogenous and weakly stratified flows. 


\subsection{Numerical Studies}

As the state of computer technology is ever advancing, so too is the ability of numerical models in accurately simulating fluid mechanical processes. However, it is only relatively recently that simulations relevant to the present study have been feasible.

The first such study is that of Smyth et al. (1988), where the nonlinear development of the Holmboe instability was simulated in two-dimensions. This confirmed previous predictions that the instability consisted of two cusp-like waves that travel on the density interface. Mixing in the Homboe 'waves' was observed to occur through thin wisps of fluid being drawn off the cusp areas and subsequently entrained into the upper and lower layers. However, the use of a two-dimensional model was not able to determine, whether significant mixing and turbulence was generated by the instability.

These questions were later answered, in part, by the recent findings in Smyth and Winters (2003), where mixing and turbulence in $\mathrm{KH}$ and Holmboe instabilities were examined at a single point in parameter space. The results showed that in this case the Holmboe instability is responsible for higher levels of mixing and turbulence generation than the $\mathrm{KH}$ instability. This result was rather unexpected considering the long-standing belief that the absence of significant regions of overturning precluded it from high levels of mixing (Thorpe, 1987). The low linear growth rate of the Holmboe instability was also expected to be an indicator that it did not develop levels of turbulence and mixing comparable to the $\mathrm{KH}$ instability. However, the linear growth rate may not be a reliable indicator of the mixing behavior of a particular instability, as it is only applicable to the linear growth of the unstable 
mode and says nothing of the nonlinear development. Indeed, mixing is invariably a nonlinear phenomenon.

Other important advancements in the study of mixing and turbulence in stratified shear flows were made by Caulfield and Peltier (2000). This study focused on the origin and development of secondary instabilities in homogenous and stratified $\mathrm{KH}$ billows. These were found to take the form of streamwise vortices and are expected to lead directly to the onset of turbulent motion. The origin of the streamwise vortices is dependent on the value of the bulk Richardson number, $J$, with different growth mechanisms present for cases of strong or weak stratification.

In the $\mathrm{KH}$ flows examined the onset of turbulence also marked a phase of heightened scalar mixing - a possible indication of the mixing transition (as discussed in Dimotakis (2000)). This behavior became less apparent as $J$ was increased (Peltier and Caulfield, 2003), resulting in a larger percentage of the mixing occurring in the preturbulent phase (i.e., the phase of the flow preceding the transition to turbulence). The importance of this preturbulent phase was recognized in SW03, where it was found that the majority of the mixing occurred. 


\section{Chapter 3}

\section{Background}

Many flows of a geophysical and engineering nature involve the horizontal shearing of a stably stratified density interface. Under certain conditions this flow will become unstable and develop hydrodynamic instabilities. These instabilities are responsible for leading the flow to a turbulent state whereby irreversible mixing of the density field is accomplished. The exact form that these instabilities take - and hence, the evolution of the flow - is dependent on a number of parameters describing the initial velocity and density distributions.

Studies to date have predominantly focused on the case in which the centre of the shear layer and the density interface coincide - referred to herein as the symmetric case. In this case two different instabilities are possible depending upon the relative strength of the stratification and the thickness of the density interface. When levels of stratification are sufficiently weak the Kelvin-Helmholtz (KH) instability results. The $\mathrm{KH}$ instability is characterized by a periodic roll-up of the density interface caused by the concentration of shear layer vorticity into discrete billows. The instability has the appearance of a stationary breaking wave in which the density interface curls up to form statically unstable regions that move with the mean velocity of the flow. The unstable regions of the billow continue to rollup until a saturated amplitude is reached. During this phase of essentially two-dimensional development, secondary instabilities begin to grow. As the billow becomes satu- 
rated the interaction of the secondary instabilities is thought to lead the flow into a turbulent collapse, after which a relaminarization occurs (Caulfield and Peltier, 2000; Peltier and Caulfield, 2003).

As the level of stratification is increased, and the density interface is kept relatively thin in comparison to the shear layer thickness, the shear is no longer able to overturn the density interface and Holmboe instability develops (Holmboe, 1962). Holmboe instability is thought to be the result of a resonant interaction between disturbances in the shear layer vorticity and internal gravity waves on the density interface (Baines and Mitsudera, 1994). Since the density gradient is sufficiently strong and thin, it can be thought of as dividing the shear layer vorticity into two equal segments (since we are dealing with the symmetric case) that have only a limited interaction with one another. For this reason, instability develops on each side of the density interface, and is characterized by cusp-like waves that travel at equal and opposite speeds with respect to the mean flow. Each of these unstable waves is referred to as a Holmboe mode.

When asymmetry is introduced (i.e., the velocity and density profiles are offset from one another), and the density interface remains thin, linear theory suggests that one of the two unstable Holmboe modes become preferentially destabilized while the other is more strongly stabilized (Lawrence et al., 1991). This causes the phenomenon of 'one-sidedness', where disturbances and mixing are found to occur on only one side of the density interface. The occurrence of one-sided flows has been noted in the tilting tube experiments of Thorpe (1968), and mixing layer experiments discussed in Maxworthy and Browand (1975). In the case of mixing layer experiments, the asymmetry was found to be caused by the different boundary 
layer thicknesses between the high- and low-speed layers (Lawrence et al., 1998). This results in a greater amount of vorticity in the high-speed layer, and a onesidedness in the development of the instabilities.

In addition to mixing layer facilities, the effects of asymmetry have been observed in a number of flows of considerable oceanographic importance. Most notably, in the exchange flow that occurs over the Camarinal Sill in the Strait of Gibraltar (Farmer and Armi, 1998). Here high salinity water from the Mediterranean Sea meets lower salinity Atlantic water, and a bi-directional exchange flow is set up in which an asymmetry is observed in the measured velocity and density profiles. Using acoustic imaging techniques, the presence of a variety of instabilities resembling $\mathrm{KH}$ billows were discovered. The presence of instabilities in flows of this nature have been found to significantly alter their dynamics (e.g.: Winters and Seim (2000); Farmer and Armi (1998)).

One-sidedness is also a common feature in the dynamics of stratified rivers, where dense salt water meets the lighter, fresh water from the river discharge. This is known to be the case in the study of salt-wedge intrusions by Sargent and Jirka (1987), Yonemitsu et al. (1996) and Yoshida et al. (1998). In the latter of these studies a one-sided overturn was observed using acoustic imaging in the Ishikari River. The overturn is expected to be the result of asymmetry in the velocity and density distributions as well as asymmetrically placed vertical boundaries.

Asymmetric density stratified shear instability constitutes a largely unexplored area in the study of mixing in shear flows. To date, the majority of studies have focused on homogenous and stratified free shear layers subject to the $\mathrm{KH}$ instability (see Thorpe (1968); Breidenthal (1981); Koochesfahani and Dimotakis (1986); 
Caulfield and Peltier (2000)). This likely stems from the long-standing belief that the $\mathrm{KH}$ instability is the only shear instability to develop a complete overturning of the density interface, precluding other instabilities as a significant source of mixing (Thorpe, 1987). However, the recent findings of SW03 indicate that in certain regions of parameter space the Holmboe instability, if given enough time, is able to generate levels of mixing and turbulence that exceed those of the $\mathrm{KH}$ instability. The long-term nonlinear evolution and mixing behavior of $\mathrm{AH}$ instabilities have not presently been studied.

In this chapter, the results of SW03 are extended to the asymmetric case, where the evolution of the turbulent transition and mixing behavior are examined for a variety of asymmetries. This is done through a series of direct numerical simulations. In the remainder of this section an introduction is given to relevant parameters, and the framework by which the evolution of the flow is studied. Chapter 4 focuses on details of the numerical solution method used. This is followed by a description of the simulation results in chapter 5 , a discussion in chapter 6 , and conclusions and future work in chapter 7 .

\subsection{Relevant Parameters}

The basic components of a density stratified shear layer consist of initial velocity and density profiles whose variation in the vertical will be represented by hyperbolic tangent functions. A schematic of these idealized profiles is shown in figure 3.1. The stable, layered density distribution has a total difference in density of $\Delta \rho$, and varies over a length scale $\eta$ between the two layers. Similarly, the velocity difference between the two streams is represented by $\Delta u$, which varies continuously over a 


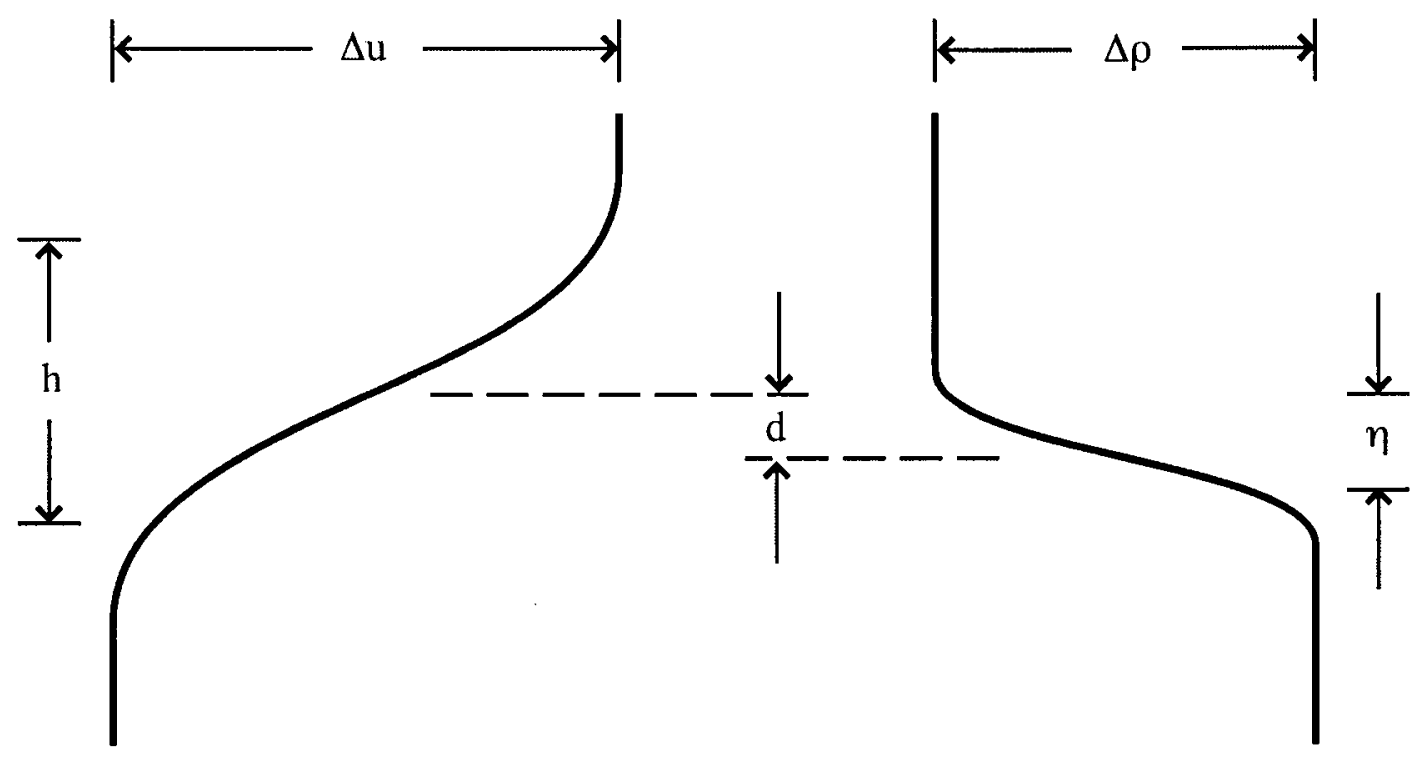

Figure 3.1: Schematic of velocity and density distribution parameters. 
length scale $h$. The offset between the centre of the shear layer and the centre of the density interface is denoted by $d$. From these variables it is possible to define two important dimensionless parameters to the evolution of the flow. These consist of the scale ratio $R=h / \eta$, and the asymmetry factor $\epsilon=2 d / h$. They measure the relative thickness of the velocity and density variations, and the magnitude of the profile asymmetry, respectively. These profiles are represented mathematically by

$$
U(z)=\frac{\Delta u}{2} \tanh \left(\frac{2}{h} z-\epsilon\right)
$$

and

$$
\bar{\rho}(z)=\frac{\Delta \rho}{2} \tanh \frac{2}{h} R z
$$

where the velocity field in a Cartesian coordinate system given by $\vec{u}=(u, v, w)$ has the initial velocity profile $\vec{u}=(U(z), 0,0)$, and the initial density profile $\bar{\rho}(z)$. Here $(x, y, z)$ denote the streamwise, spanwise, and vertical directions, respectively.

Although these profiles are an idealization, the use of hyperbolic tangent profiles was tested experimentally in the laboratory investigation of Yonemitsu et al. (1996), where a good agreement was found in the case of salt-wedge intrusions; a flow known to exhibit asymmetry (see $\S 3)$.

If we now consider a stratifying agent (usually either heat or salt) with a molecular diffusivity of $\kappa$, in a fluid of kinematic viscosity $\nu$, and a reference density of $\rho_{0}$, three additional dimensionless parameters result:

$$
R e=\frac{\Delta u h}{\nu} ; \quad J=\frac{\Delta \rho g h}{\rho_{0}(\Delta u)^{2}} ; \quad \operatorname{Pr}=\frac{\nu}{\kappa}
$$

The Reynolds number, Re, for the simulations discussed in this thesis is taken as 
1200. This value is more representative of laboratory experiments but also includes low-Re mixing events expected in oceans and lakes (Thorpe, 1985). Notably, this $R e$ is the same order of magnitude as the instabilities observed in the seasonal thermocline of the Mediterranean Sea by Woods (1968). The bulk Richardson number, $J$, represents the ratio of the stabilizing effect of stratification to the destabilizing effect of the velocity shear, and is chosen to be 0.15 . This value of $J$ allows for the growth of both $\mathrm{KH}$ and Holmboe instabilities through an adjustment of the scale ratio $R$, and is representative of conditions found in both field and laboratory studies (Thorpe, 1985). The Prandtl number, $P r$, is the ratio of the kinematic viscosity to the molecular diffusivity of the stratifying agent, and is therefore a property of the fluid. A value of $\operatorname{Pr}=9$ was chosen for the current simulations which corresponds to thermal stratification in both fresh and salt water. It should be noted that $\operatorname{Pr}$ is also used as a guide in the choice of the initial value of $R$. This can easily be seen by considering only the process of diffusion acting on both the vorticity of the shear layer, and the stratifying agent of the density interface. Given a time scale $\tau$, each interface will grow by diffusion according to $h \sim(\nu \tau)^{1 / 2}$ and $\eta \sim(\kappa \tau)^{1 / 2}$. Taking the ratio of these length scales $R \sim P r^{1 / 2}$ is found. Therefore, in most circumstances $R=\operatorname{Pr}^{1 / 2}$ will be taken.

Choice of the parameters mentioned above was also motivated by numerical constraints (to be discussed further in section 4.1) and to facilitate better comparison to the results of the simulations performed in SW03. 


\subsection{The Partition and Transfer of Energy}

To gain insight into the processes involved in the evolution of the flow through its various stages of development it is useful to partition the energy into a number of different reservoirs. As the flow evolves, the energy transfers between these reservoirs are calculated based on the framework of Winters et al. (1995). This begins with the definition of the average total potential energy, expressed in dimensionless units as

$$
P \equiv \frac{g}{(\Delta u)^{2} \rho_{0}}\langle\rho z\rangle_{V}
$$

where $\langle\cdot\rangle_{V}$ denotes a volume average over the computational domain, and $g$ is the gravitational acceleration. $P$ can now be partitioned into the available potential energy, $P_{A}$, and background potential energy, $P_{B}$, reservoirs. Here $P_{B}$ is defined as

$$
P_{B} \equiv \frac{g}{(\Delta u)^{2} \rho_{0}}\left\langle\rho_{B} z\right\rangle_{V}
$$

where $\rho_{B}=\rho_{B}(z)$ is the background density profile. This profile represents the minimum potential energy state of the density field if fluid elements are rearranged and deformed adiabatically, i.e., without altering their density. Such a profile is a monotonically decreasing function of height that is statically stable everywhere. The significance of $\rho_{B}$ lies in the fact that for a closed domain any changes to this profile are irreversible, and lead to an increased $P_{B}$ as time proceeds (Winters et al., 1995). These changes are closely linked with the rate of mixing of the density field. It is now possible to define $P_{A}$ by

$$
P_{A} \equiv P-P_{B}
$$


This quantity represents the amount of potential energy that is readily available to be converted back to kinetic energy to drive fluid motions. Unlike the $P_{B}$ reservoir, changes in $P_{A}$ are reversible and represent 'stirring' processes (Peltier and Caulfield, 2003). These relationships can be seen more clearly by looking at the evolution equations for the kinetic and potential energy reservoirs. For a closed domain in dimensionless units (see Winters et al. (1995) and Peltier and Caulfield (2003)):

$$
\begin{gathered}
\frac{d K}{d t}=-J\left\langle\rho_{*} w_{*}\right\rangle_{V}-R e^{-1}\left\langle\vec{u}_{*} \cdot \nabla_{*}^{2} \vec{u}_{*}\right\rangle_{V}=H-D \\
\frac{d P}{d t}=-J\left\langle\rho_{*} w_{*}\right\rangle_{V}+\frac{J}{\operatorname{RePr} L_{z}^{*}}=-H+D_{p}
\end{gathered}
$$

where the volume averaged kinetic energy is given by

$$
K=\frac{\langle\vec{u} \cdot \vec{u} / 2\rangle_{V}}{(\Delta u)^{2}}
$$

and $L_{z}^{*}$ is the dimensionless domain height. The variables and operators with an asterisk as subscript have been nondimensionalized by the velocity, length, density, and time scales given by $\Delta u, h, \Delta \rho$, and $h / \Delta u$, respectively. (Here we have chosen the notation of Caulfield and Peltier (2000) and Peltier and Caulfield (2003) as much as possible.)

The buoyancy flux, $H$, represents the reversible transfer of energy between the kinetic and potential energy reservoirs. This can be seen by its presence in (3.8), where it is of opposite sign, indicating that transfers exist between both reservoirs. Here we have used the convention of Peltier and Caulfield (2003) where $H>0(H<$ 0 ) represents the lifting (sinking) of lighter fluid parcels, and leads to a corresponding decrease (increase) in $P$. The second term in (3.7), $D$, gives the average loss of 
kinetic energy due to viscous dissipation. This constitutes an irreversible transfer from $K$ to the internal energy reservoir of the fluid. Since $D$ acts most effectively on the small scales of velocity variation it will be used as an indicator for the level of turbulence present in the flow. The last term in (3.8), $D_{p}$, represents the irreversible rate of increase of $P$ due to the molecular diffusion of the initial density profile. In this representation it has been assumed that $\bar{\rho}_{\text {bottom }}-\bar{\rho}_{\text {top }}=\Delta \rho$, where $\bar{\rho}_{\text {bottom }}$ and $\bar{\rho}_{t o p}$ denote the average density on the bottom and top vertical domain boundaries, respectively. This assumption holds for sufficiently large domain heights such that the average density at the vertical domain boundaries remains constant, and the difference is always $\Delta \rho$. This term plays no active role in the dynamics of the flow but serves to quantify the rate of increase in $P$ that occurs in the absence of fluid motion.

In order to quantify the rate of fluid mixing similar equations can be written for $P_{A}$ and $P_{B}$ as follows:

$$
\begin{aligned}
& \frac{d P_{A}}{d t}=-H-M \\
& \frac{d P_{B}}{d t}=M+D_{p} .
\end{aligned}
$$

Here $M$ represents the instantaneous mixing rate, defined as the rate of increase in $P_{B}$ due solely to fluid motions (Peltier and Caulfield, 2003). Since changes in $P_{B}$ can be computed quite easily by a simple sorting of the density field, this quantification of the mixing rate lends itself well to the numerical simulations performed here. The interaction between various energy reservoirs described by evolution equations (3.7) - (3.11) are shown schematically in figure 3.2. The partition of $P$ into $P_{A}$ and $P_{B}$ allows for the distinction between stirring and mixing processes. The stirring 


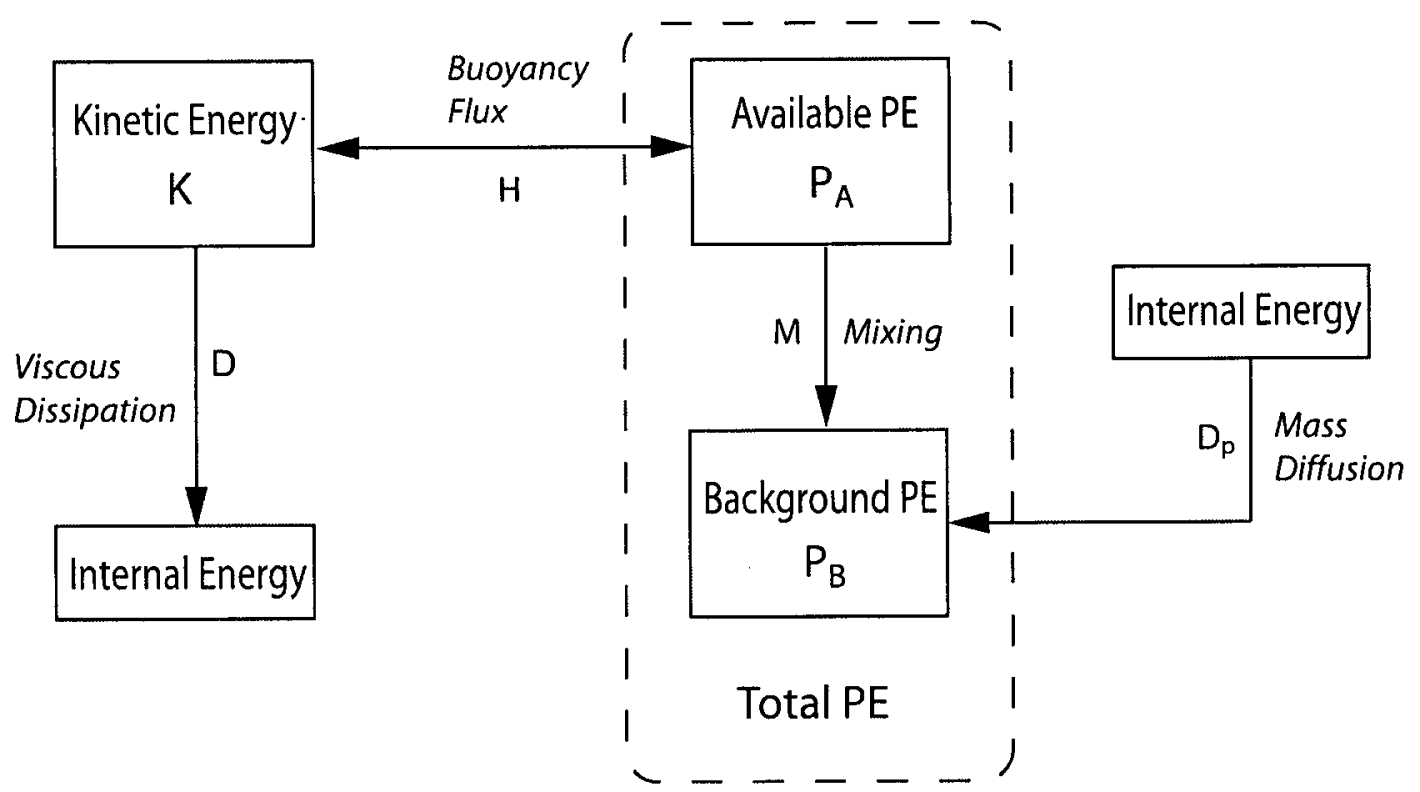

Figure 3.2: Illustration of the relationships between the various energy reservoirs (after Winters et al. (1995)). 
processes being associated with reversible energy transfers to and from $P_{A}$, and mixing processes being due exclusively to the irreversible transfer of energy to $P_{B}$ caused by fluid motion.

It is also instructive to partition $K$ into components that can be associated with the kinetic energy of motions related to the mean flow, the two-dimensional primary instability, and the three-dimensional secondary instabilities and subsequent turbulence. Following Caulfield and Peltier (2000), this is done as follows:

$$
\begin{gathered}
\bar{K}=\frac{1}{(\Delta u)^{2}}\left\langle\left(\bar{u}^{2}\right) / 2\right\rangle_{z} \\
K_{2 d}=\frac{1}{(\Delta u)^{2}}\left\langle\left(\vec{u}_{2 d} \cdot \vec{u}_{2 d}\right) / 2\right\rangle_{y z} \\
K_{3 d}=\frac{1}{(\Delta u)^{2}}\left\langle\left(\vec{u}_{3 d} \cdot \vec{u}_{3 d}\right) / 2\right\rangle_{x y z}
\end{gathered}
$$

where

$$
\begin{gathered}
\bar{u}(z, t)=\langle\vec{u}\rangle_{x y}, \\
\vec{u}_{2 d}(x, z, t)=\langle\vec{u}-\bar{u}(z)\rangle_{y}, \\
\vec{u}_{3 d}(x, y, z, t)=\vec{u}-\bar{u}-\vec{u}_{2 d} .
\end{gathered}
$$

From these definitions it can be seen that

$$
K=\bar{K}+K_{2 d}+K_{3 d}
$$

It is important to note that the exchange between these reservoirs is reversible, implying that energy may be transfered directly from $P_{A}$ via $H$ to any of the kinetic energy reservoirs. 


\subsection{Mixing Efficiency}

In the study of stratified flows, the efficiency of a mixing event has traditionally been defined as the ratio of the increase in potential energy of the system to the work done by the kinetic energy of the driving mechanism (Caulfield and Peltier, 2000). In this case, kinetic energy is extracted from the shear layer, and work is done by the instability on the fluid within the computational domain through mixing of the density field, and in losses due to viscous dissipation. With this in mind, a suitable measure of the mixing efficiency can be arrived at by inspection of the energy evolution equations (3.7) - (3.11). First, note that if a hypothetical reservoir is defined that consists of both $K$ and $P_{A}$ then the reversible transfer associated with $H$ is eliminated and all transfers between reservoirs are irreversible. Ignoring $D_{p}$, since it plays no active role in mixing, it can be seen that the energy consumed by the mixing event can be used for two different processes: to be lost to the internal energy of the fluid by viscous dissipation, or to be used to mix the density field and supply background potential energy. So by defining the instantaneous mixing efficiency, $E_{i}$, as

$$
E_{i} \equiv \frac{M}{M+D}
$$

we are taking the ratio of the rate at which the mixing event is supplying potential energy, to the total rate at which energy is being extracted in the process. This definition is equivalent to that discussed in Caulfeld and Peltier (2000) and Peltier and Caulfield (2003), and has the property $0<E_{i}<1$.

Of course $E_{i}$ alone is not sufficient to describe the mixing behavior in a particular flow - the magnitude of the energy transfers are also important. For example, if 
both $M$ and $D$ are small then $E_{i}$ may be close to unity indicating highly efficient mixing, yet the transfers themselves do not represent a significant mixing process.

It is also useful to define a cumulative mixing efficiency as

$$
E_{c} \equiv \frac{\int_{0}^{T} M d t}{\int_{0}^{T} M d t-\int_{0}^{T} D d t}
$$

where $T$ denotes some duration of interest (Peltier and Caulfield, 2003). If $T$ is taken as the total duration of a mixing event then $E_{c}$ gives the total proportion of energy that is used to perform mixing to the total amount of energy expended in the process. This gives an indication of the mixing efficiency of the entire event, rather than an instantaneous value. 


\section{Chapter 4}

\section{Numerical Solution Method}

\subsection{Direct Numerical Simulations}

The numerical simulations performed in this study were carried out using a threedimensional spectral model designed for the study of stratified flows (see Winters et al. (2004) for a full discussion). It is used here to solve the incompressible equations of motion for a Boussinesq fluid in the absence of external forces and rotational effects described by

$$
\frac{D \vec{u}}{D t}=-\frac{1}{\rho_{0}} \nabla p-\frac{\rho}{\rho_{0}} g \hat{k}+\nu \nabla^{2} \vec{u}
$$

and

$$
\nabla \cdot \vec{u}=0
$$

Here $D / D t=\partial / \partial t+\vec{u} \cdot \nabla$ denotes a material derivative, $\hat{k}$ is the unit vector in the vertical direction (positive upwards), and $\vec{u}=(u, v, w)$ represents the velocity field in the streamwise, spanwise, and vertical directions, respectively. The pressure field is given by $p$, and the density field by $\rho$. Since the density field is governed by an active scalar it evolves subject to the advection-diffusion equation

$$
\frac{D \rho}{D t}=\kappa \nabla^{2} \rho
$$


For the purposes of this study any nonlinearities in the equation of state are neglected.

Since the viscous and diffusive terms from (4.1) and (4.3) are solved directly without any turbulence closure scheme this numerical solution method fits into the direct numerical simulations (DNS) category. Due to the present limitations involving computational resources, DNS is constrained to study flows with relatively small domain sizes, and lower values of $R e$ and $P r$ than would often occur in nature.

In the DNS of a homogenous fluid the difficulty of simulating high Re flows lies in the large range of length scale variability, where the largest scales are generally given by the domain size, and the smallest scales are $O\left(L_{K}\right)$ (Moin and Mahesh, 1998). Here, $L_{K}=\left(\nu^{3} / \varepsilon\right)^{1 / 4}$ is the Kolmogorov length scale, where $\varepsilon$ is the rate of viscous dissipation. This represents the scale at which velocity gradients are diffusively smoothed by viscosity. However, in a stratified flow where $\operatorname{Pr}>1$, as is the case here, the smallest scales are determined by the Batchelor length $L_{B}=L_{K} / \operatorname{Pr}^{1 / 2}$ (Batchelor, 1959). This expresses the fact that the smallest scales are now determined by scalar gradients smoothed by molecular diffusion - a relatively slower process. High $\operatorname{Pr}$ fluids are therefore able to generate significantly smaller scales than realized in otherwise homogenous flows.

This limitation has been partially alleviated by a modification of the Winters et al. (2004) model by W.D. Smyth to aid in the simulation of higher $\operatorname{Pr}$ fluids. The modification includes an additional active scalar field that is resolved on a grid twice as fine as the velocity and pressure grids. Originally designed for the study of differential diffusion, i.e., the mixing of two scalar fields with different $\operatorname{Pr}$ (see Smyth et al. (2004)), it is used here for a single scalar at moderate Pr. 


\subsection{Initial and Boundary Conditions}

In order to reduce the number of time steps required for the initial growth of the primary instability a perturbation is applied to the initial velocity and density fields. The perturbation varies only in the streamwise direction (as the primary instability is two-dimensional, confirmed by Haigh (1995)) and is determined from the solution of the Taylor-Goldstein equation incorporating the effects of viscosity and mass diffusion.

Solution of the Taylor-Goldstein equation predicts the structure of the resulting instability, given by the eigenfunctions, as well as the growth rate and phase speed for a disturbance of a given wavenumber, as found in the eigenvalues. Since it is generally found that the wavenumber of the fastest growing mode dominates the nonlinear development of the flow (Lawrence et al. (1991)), the perturbation is applied at the wavenumber of maximum growth rate. This solution is based on linear theory and assumes that the perturbations have infinitesimally small amplitude. For this reason, the perturbation must be small enough to ensure it spends some time in the linear regime and not adversely affect the growth of the instability. A maximum displacement amplitude of $0.2 h$ was found to be sufficient for these purposes.

In addition to the perturbation described above, a random perturbation is applied to the velocity field to stimulate the growth of three-dimensional secondary instabilities. The amplitude of the perturbation is evenly distributed between $\pm 0.01 \Delta u$, and is centred on the density interface.

The computational domain consists of lengths in each of the streamwise, spanwise, and vertical directions denoted by $\left\{L_{x}, L_{y}, L_{z}\right\}$, respectively. The boundary conditions are periodic in both the streamwise and spanwise directions so that $L_{x}$ is 


\begin{tabular}{cccccccc}
\multicolumn{7}{c}{ Simulation } \\
\hline & 1 & 2 & 3 & 4 & 5 & 6 & 7 \\
\hline$\epsilon$ & 0 & 0 & 0.25 & 0.50 & 1.0 & 1.5 & 2.0 \\
$J$ & 0.15 & 0.15 & 0.15 & 0.15 & 0.15 & 0.15 & 0.15 \\
$R e$ & 1200 & 1200 & 1200 & 1200 & 1200 & 1200 & 1200 \\
$P r$ & 9 & 9 & 9 & 9 & 9 & 9 & 9 \\
$R$ & 1 & 3 & 3 & 3 & 3 & 3 & 3 \\
$N_{x}$ & 128 & 128 & 128 & 128 & 128 & 128 & 128 \\
$N_{y}$ & 96 & 64 & 96 & 96 & 96 & 96 & 96 \\
$N_{z}$ & 192 & 128 & 192 & 192 & 192 & 192 & 192 \\
$L_{x} / h$ & 6.98 & 7.85 & 6.16 & 5.71 & 5.71 & 6.04 & 6.28 \\
$L_{y} / h$ & 4.50 & 4.50 & 4.50 & 4.50 & 4.50 & 4.50 & 4.50 \\
$L_{z} / h$ & 9.00 & 9.00 & 9.00 & 9.00 & 9.00 & 9.00 & 9.00 \\
$\sigma$ & 0.081 & 0.025 & 0.090 & 0.131 & 0.163 & 0.172 & 0.176
\end{tabular}

Table 4.1: Summary of numerical simulations performed.

given by the fastest growing wavelength determined by the linear stability analysis. The streamwise extent, therefore, varies for each simulation. In order to accommodate a number of wavelengths of the resulting secondary instabilities all simulations have $L_{y}=4.5 h$. This estimate is based on the observations of secondary instabilities from KH and Holmboe simulations in Caulfield and Peltier (2000) and SW03. The no-flux, free-slip boundary conditions are employed in the vertical directions requiring the vertical velocity and vertical fluxes to vanish, i.e.,

$$
\left.w\right|_{z=0, L_{z}}=\left.\frac{\partial u}{\partial z}\right|_{z=0, L_{z}}=\left.\frac{\partial v}{\partial z}\right|_{z=0, L_{z}}=0,\left.\quad \frac{\partial \rho}{\partial z}\right|_{z=0, L_{z}}=0
$$

A value of $L_{z}=9 h$ was chosen so that the vertical boundaries are large enough to have a negligible influence on the development of the instability. A table summarizing the simulations discussed in this chapter is shown in table 4.1. Here $\left\{N_{x}, N_{y}, N_{z}\right\}$ correspond to the number of grid points used in each of the component directions 
in the coarser velocity fields, and $\sigma$ is the exponential growth rate of the instability determined from linear theory. 


\section{Chapter 5}

\section{Results}

\subsection{The Symmetric Case: $\mathrm{KH}$ and Holmboe Instabilities}

Simulation results are examined first in the symmetric case $(\epsilon=0)$, where $\mathrm{KH}$ and Holmboe instabilities are found to develop. This gives a basis for comparison in the asymmetric cases $(\epsilon \neq 0)$, and helps illustrate the effects that asymmetry has on the flow.

\section{Kelvin-Helmholtz case:}

Figure 5.1 shows the evolution of the potential energy reservoirs, the irreversible transfers $M$ and $D$, and $E_{i}$ for the $\mathrm{KH}$ instability (as performed in SW03 using a slightly different mixing efficiency and domain size). In figure 5.1, the dotted line represents the potential energy gain resulting from diffusion of the initial profile, obtained from

$$
\Phi(t)=\int_{0}^{t} D_{p}\left(t^{\prime}\right) d t^{\prime} .
$$

This is done for comparison purposes with $P$ and $P_{B}$, given by the solid and dashed lines respectively. The KH curves show a relatively rapid growth in $P_{A}$ (obtained from the difference between $P$ and $P_{B}$ ) due to the roll-up of the primary billow. 

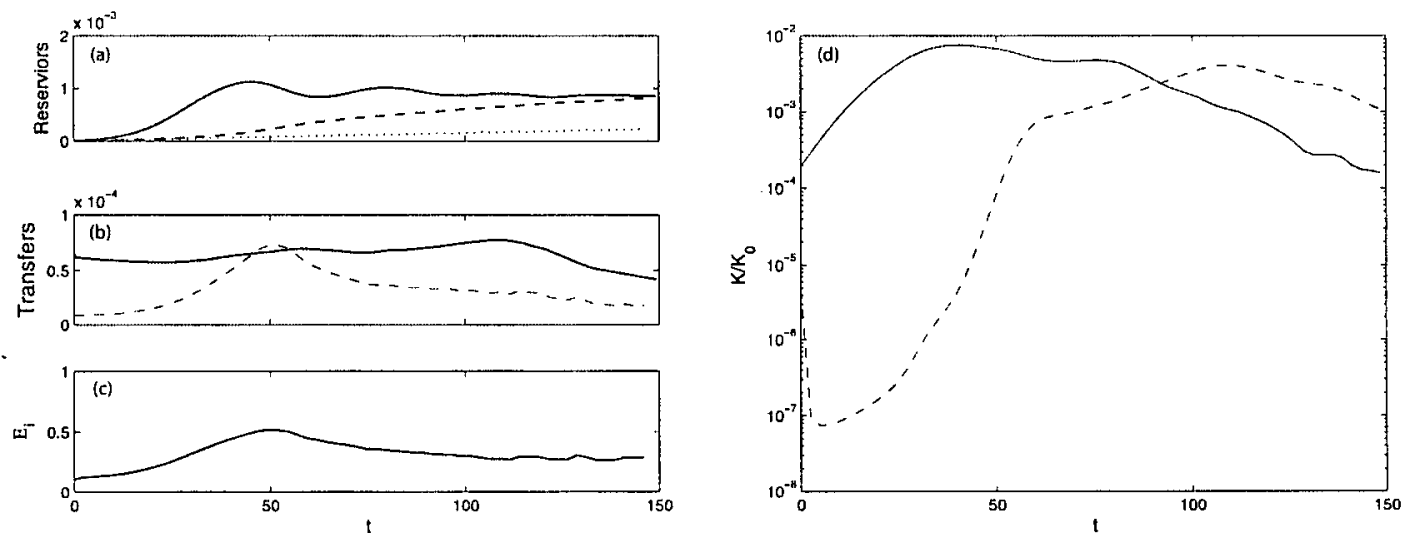

Figure 5.1: Energy reservoirs and transfers for the KH simulation. The plot in (a) includes the potential energy reservoirs $P$ (solid line), $P_{B}$ (dashed line), and $\Phi$ (dotted line). In (b) both irreversible transfers $D$ (solid line), and $M$ (dashed line) are shown along with $E_{i}$ in (c). The $K_{2 d}$ (solid line) and $K_{3 d}$ (dashed line) are plotted in (d).

This can be seen in the two-dimensional $x z$-slices of the density field shown in figure 5.2. These slices are taken at $y=L_{y} / 2$ and are similar to visualizations of the density field encountered in laboratory investigations where light sheets are used to illuminate the flow (e.g.: Lawrence et al. (1991); Schowalter et al. (1994); Hogg and Ivey (2003)).

At $t \approx 45$ the flow has reached its state of maximum potential energy (and maximum $P_{A}$ ) when the billow has saturated. Shortly after this time the billow core begins to collapse owing to the growth of three-dimensional secondary instabilities associated with the locally unstable regions around the billow core. Thus far the rate of mixing has steadily increased until its peak at $t \approx 50$. At this time the secondary instabilities have evolved into coherent structures within the billow core. These structures resemble those observed in the stratified mixing layer experiments of Schowalter et al. (1994). However, the flow within the billow (or elsewhere) 
0

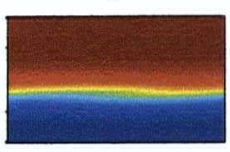

57

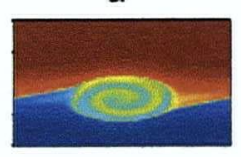

116

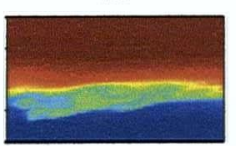

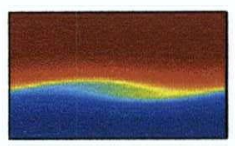

74

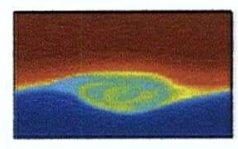

129

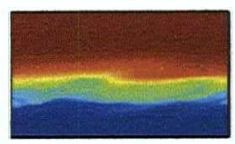

30

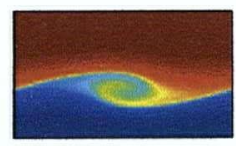

87

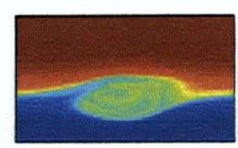

144

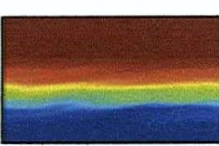

45

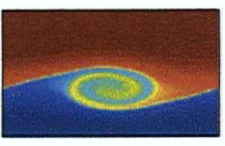

101

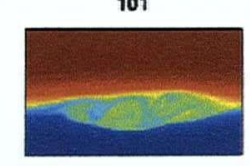

Figure 5.2: Plots of the density field in the $\mathrm{KH}$ simulation showing its time evolution. Slices of the $x z$-plane are taken at $y=L_{y} / 2$ with times shown in each plot. 
cannot be called turbulent; but, on the verge of turbulent collapse. As can be seen in figure 5.2, the greater degree of mixing within the core region has produced a 'pocket' of intermediate density fluid that is vertically centred between the two relatively undisturbed layers. Through a combination of both the buoyancy forces and the background shear, this pocket spreads laterally throughout the centre of the domain where the density profile assumes a statically stable configuration as the flow relaminarizes.

By inspection of figure $5.1 \mathrm{~b}$, it can be seen that the highest levels of viscous dissipation occur well after the highest levels of mixing, and can be identified with the lateral spreading and shearing of the mixed billow core. In this stage of the flow, the surplus of $P_{A}$ gained initially by the billow roll-up has largely been consumed. With no other stirring mechanism present for the formation of $P_{A}$ the small-scale turbulent motions have little effect on mixing. This general trend can be seen in figure $5.1 \mathrm{c}$, where $E_{i}$ peaks in the early stages of 'preturbulent mixing', while the later stages are marked by a much lower efficiency corresponding to the spreading and shearing of the mixed billow core.

\section{Symmetric Holmboe case:}

As the scale ratio $R$, is increased, the stratification is compressed to a layer thinner than the shear thickness. While $J$ remains unchanged, the density gradient is locally sharpened in the centre of the domain. This results in the formation of Holmboe's instability as seen in figure 5.3. The growth of Holmboe's instability is considerably slower than that of the $\mathrm{KH}$, as is expected from its smaller linear growth rate (see table 4.1 for a listing of the linear growth rate $\sigma$, for each simulation). Before the 
0

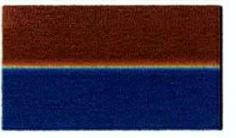

59

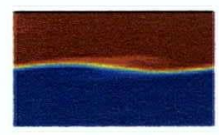

119

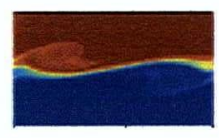

170

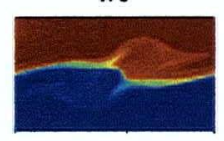

22

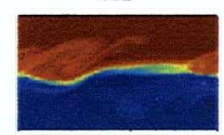

15

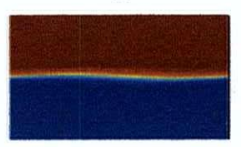

74

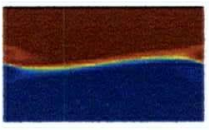

128

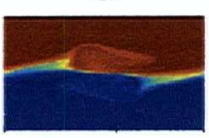

185

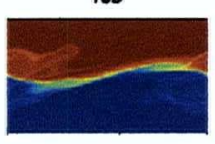

237

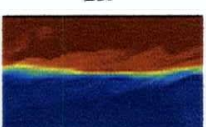

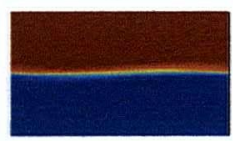

99

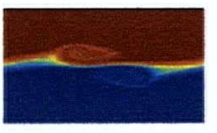

143

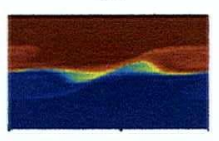

198

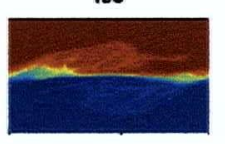

252

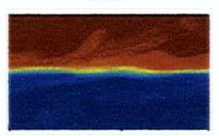

44

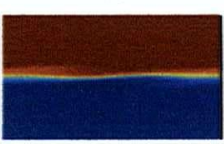

104

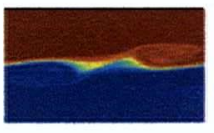

156

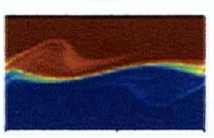

207

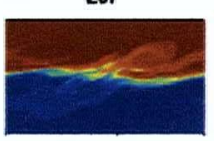

267

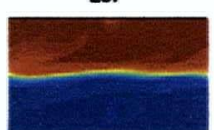

Figure 5.3: Plots of the density field in the symmetric Holmboe simulation showing its time evolution. Slices of the $x z$-plane are taken at $y=L_{y} / 2$ with times shown in each plot. 
onset of turbulent motions at $t \approx 190$ the growth of $P_{A}$ and $K_{2 d}$ can be seen in figure $5.4 \mathrm{a}$ and $5.4 \mathrm{~b}$ to be marked by a pronounced oscillation. This is due to a standing wave-like motion that develops during the growth of the instability. As was shown
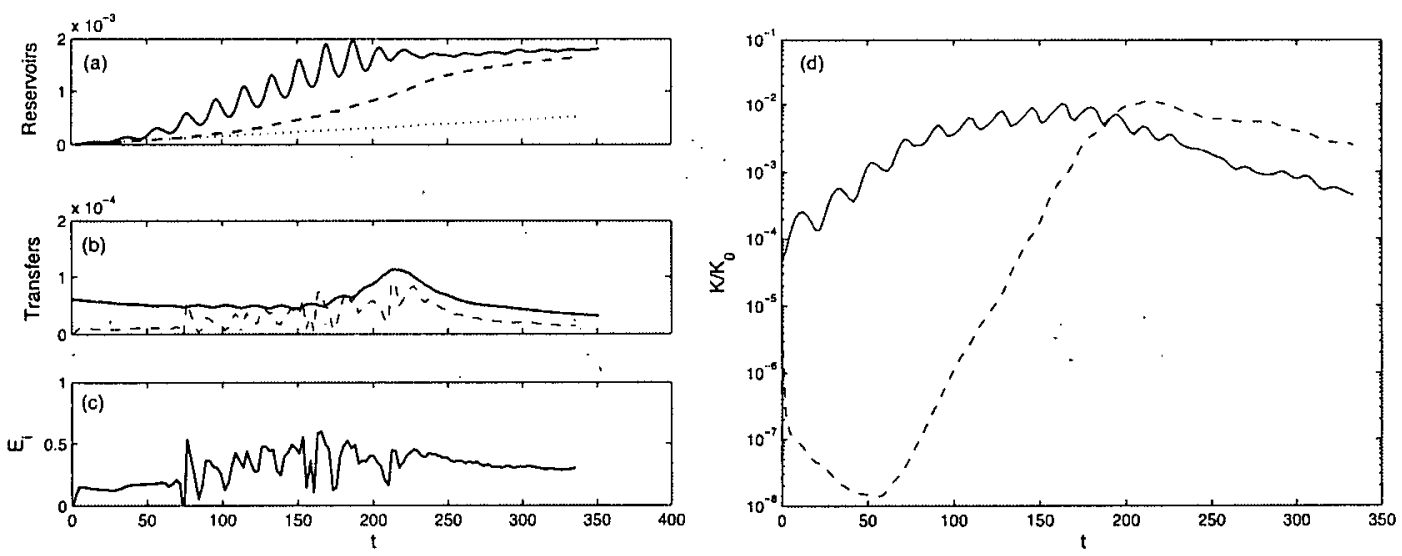

Figure 5.4: Energy reservoirs and transfers for the Holmboe simulation. The plot in (a) includes the potential energy reservoirs $P$ (solid line), $P_{B}$ (dashed line), and $\Phi$ (dotted line). In (b) both irreversible transfers $D$ (solid line), and $M$ (dashed line) are shown along with $E_{i}$ in (c). The $K_{2 d}$ (solid line) and $K_{3 d}$ (dashed line) are plotted in $(\mathrm{d})$.

in SW03, both the mixing and viscous dissipation rates (figure 5.4b) attain higher levels than the $\mathrm{KH}$. Here the rate of mixing in figure $5.4 \mathrm{~b}$ exhibits a strong variability that was not present in all other simulations to the same extent. As suggested by the preliminary results of an additional simulation at a finer resolution, the density field is not well resolved on the scale necessary for accurate computation of $M$. However, both the velocity field and the bulk mixing characteristics of the flow (taken over the entire duration of the simulation) appear to be accurate. The ill-resolved behavior of $M$ carries over to the computation of $E_{i}$ in figure $5.4 \mathrm{c}$.

The low growth rate of the Holmboe instability can be seen to postpone the 
development of secondary instabilities (indicated by $K_{3 d}$ ) until the finite amplitude waveforms develop at $t \approx 60$. Once the growth of $K_{3 d}$ begins, it is at a lower rate than the $\mathrm{KH}$ simulation and is not influenced by the standing wave motion that develops. At no point in the simulation are large-scale overturning motions present. The mixing accomplished by the Holmboe instability is due to other processes that leave the density interface relatively intact. These will be discussed in the following paragraphs in the context of $\mathrm{AH}$ instabilities as well.

\subsection{The Asymmetric Case: AH Instabilities and the Effects of Asymmetry}

A series of simulations are now presented in which the asymmetry factor, $\epsilon$, is gradually increased while leaving all other parameters constant (with the exception of $L_{x}$ as discussed in $\S 4.2$ ). Note that in these simulations $R=3$, so that we are examining $\mathrm{AH}$ instabilities that result from the relatively thin density interface, rather than asymmetric $\mathrm{KH}$ instabilities. This can also be seen from a linear stability analysis of the asymmetric configurations which shows the presence of two modes of instability: a dominant mode with a larger growth rate that forms on the side of the density interface with greater shear layer vorticity, and a weaker mode with small growth rate in the opposite layer (Lawrence et al., 1991; Haigh, 1995). However, for all simulations performed in this study the value of $J$ chosen results in the stabilization of the weaker mode according to linear theory. As can be seen in the evolution of the density fields in the following simulations, only the dominant AH mode develops initially (due in part to the initial perturbation and streamwise periodic boundary 
conditions) and grows more rapidly than both the $\mathrm{KH}$ and Holmboe instabilities, as is expected from its larger linear growth rate (cf. table 4.1).

$\epsilon=0.25$ case:

Various energy reservoirs, selected transfers, and mixing efficiency for the $\epsilon=0.25$ asymmetry are shown in figure 5.5. Initially, the potential energy of the $\epsilon=0.25$
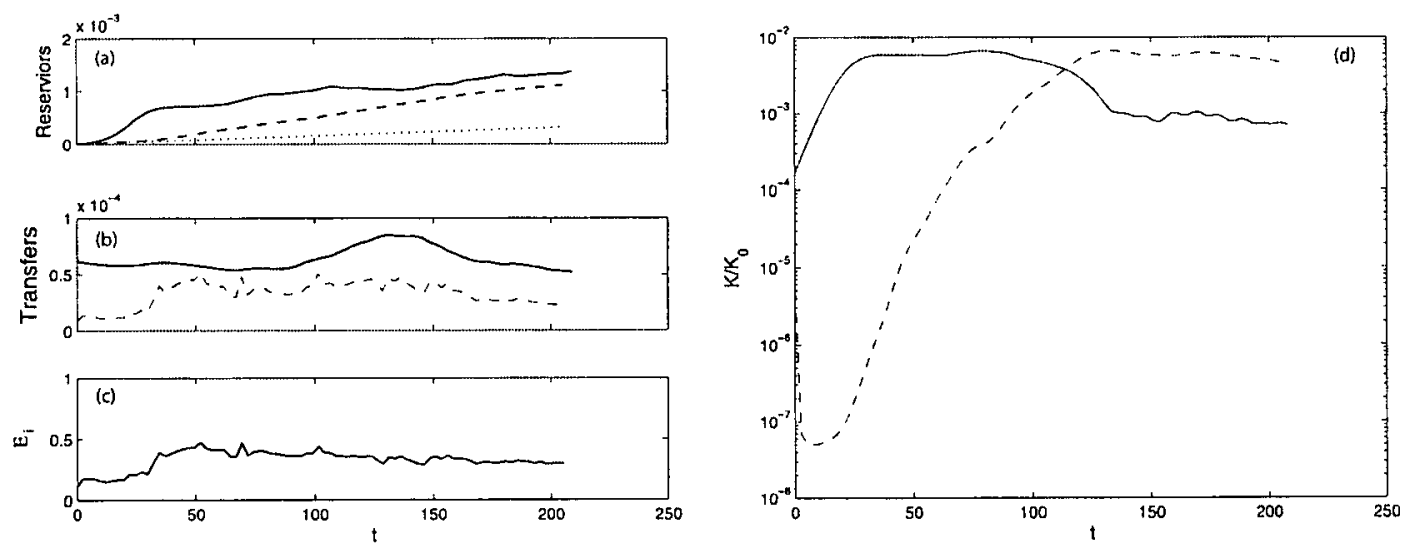

Figure 5.5: Energy reservoirs and transfers for the $\epsilon=0.25$ simulation. The plot in (a) includes the potential energy reservoirs $P$ (solid line), $P_{B}$ (dashed line), and $\Phi$ (dotted line). In (b) both irreversible transfers $D$ (solid line), and $M$ (dashed line) are shown along with $E_{i}$ in (c). The $K_{2 d}$ (solid line) and $K_{3 d}$ (dashed line) are plotted in $(d)$.

$\mathrm{AH}$ instability bears resemblance to the $\mathrm{KH}$ case, where there is a rapid growth of $P_{A}$ due to the formation of a billow structure. This billow is qualitatively different from standard $\mathrm{KH}$ billows in that it never accomplishes a complete overturning; leaving the density interface intact, it draws fluid of intermediate density from the upper portions of the interface (figure 5.6). The billow structure thins soon after its initial development to more closely resemble a Holmboe mode, where a cusp-like 

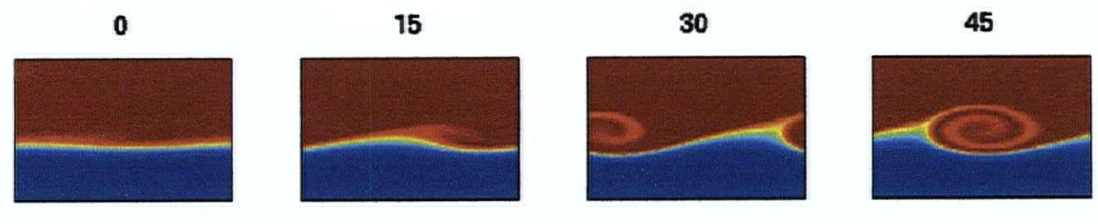

57

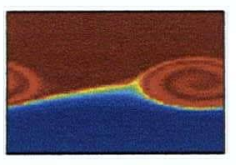

116

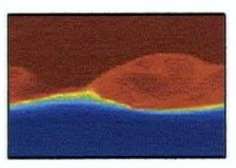

171

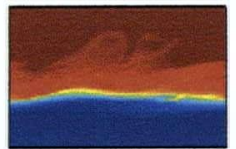

74

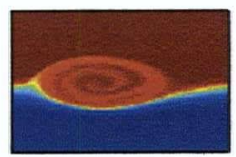

129

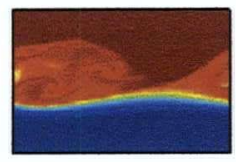

186

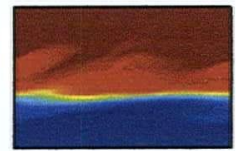

87

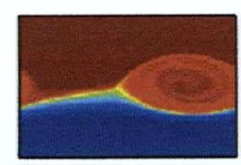

144

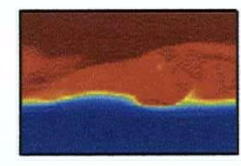

198

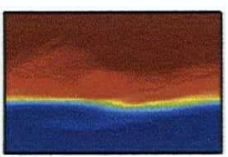

101

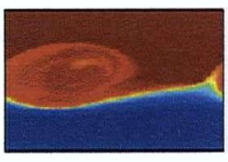

156

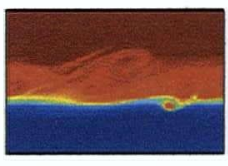

208

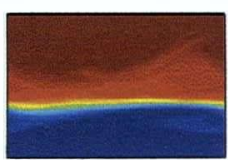

Figure 5.6: Plots of the density field in the $\epsilon=0.25$ simulation showing its time evolution. Slices of the $x z$-plane are taken at $y=L_{y} / 2$ with times shown in each plot. 
wave forms that is continually entraining a wisp of fluid into the leading vortex, located in the dominant upper layer. This entrainment process supplies $P_{A}$ which is then mixed within the primary vortex at a relatively steady rate (figure $5.5 \mathrm{~b}$ ). Mixing continues in this manner for a substantial period of time $(t \approx 45-110)$ during which secondary instabilities grow within the primary vortex.

This 'preturbulent' (to use the terminology of SW03) period shows a developing three-dimensional structure that remains coherent. As in the $\mathrm{KH}$ simulation, this coherent mixing stage is marked by the highest levels of $E_{i}$. The secondary instabilities also appear to be associated with the statically unstable regions created in the primary vortex, similar to the KH case. Figure 5.7 shows the development of this three-dimensional motion in the $y z$-plane taken from the trailing edge of the primary vortex slightly downstream of the cusp, in the later stages of the preturbulent period $(t=89)$. Density structure similar to this was observed in the mixing layer experiments of Schowalter et al. (1994) where the locally unstable stratification led to the amplification of streamwise vortices through the baroclinic generation of vorticity (see figure 26a in particular). Interaction of these streamwise vortices is thought to cause the transition to turbulent flow in KH billows (Caulfield and Peltier, 2000; Peltier and Caulfield, 2003) and appears to be in agreement with the findings here.

The breakdown of the primary vortex to incoherent three-dimensional motions at $t \approx 110$ signals a start in the rise of $D$ with a maximum at $t \approx 140$, after the majority of the mixing has occurred. This stage also results in a ceasing of the entrainment as seen in the loss of $P_{A}$ generation. However, the rate of mixing remains relatively constant, and begins to trail off only after the turbulence begins to decay and the upper layer relaminarizes. This indicates that the turbulent motions are mixing 


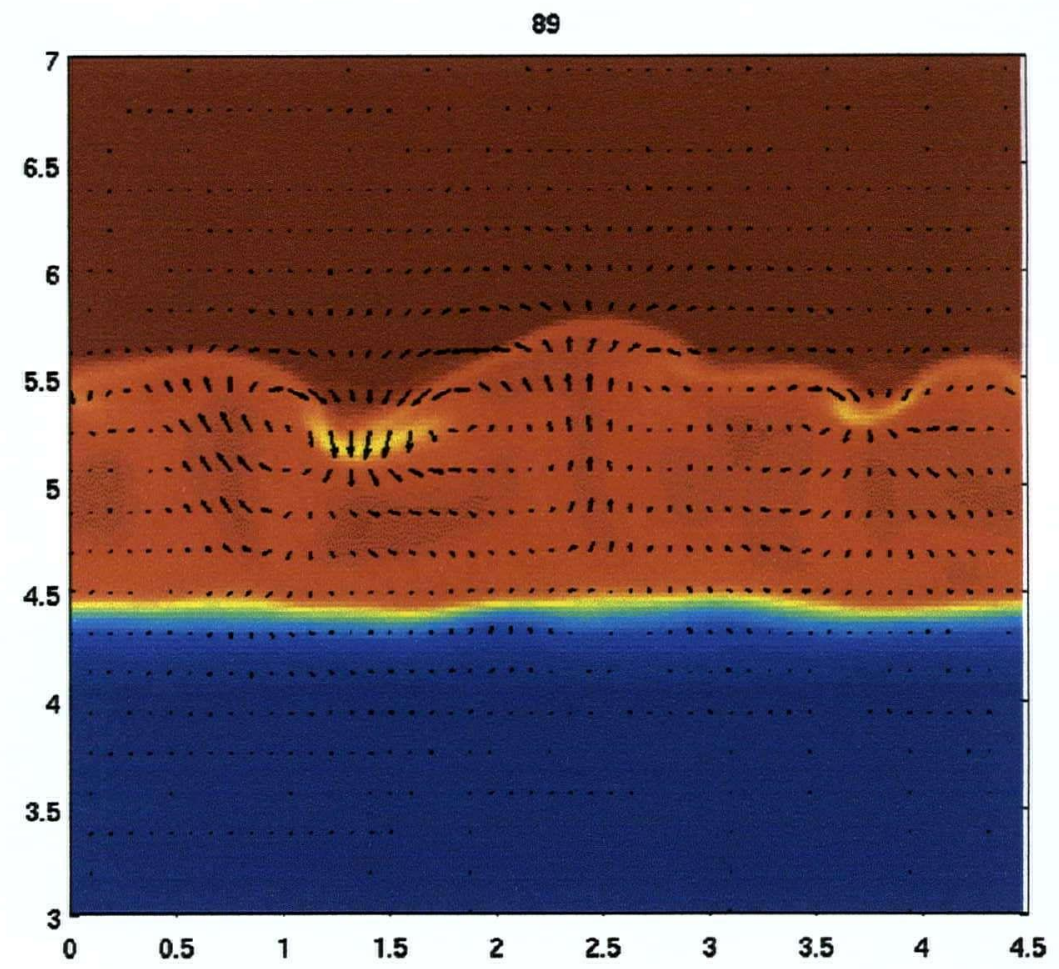

Figure 5.7: Cross-section in the $y z$-plane of the density field with $\vec{u}_{3 d}$ vectors overlaid for the $\epsilon=0.25$ simulation. The streamwise location is taken within the primary vortex for $x=4 h$ at $t=89$. 
previously entrained - but not completely mixed - fluid in the dominant layer.

It should be noted that towards the end of the simulation the weaker mode has begun to develop in the lower layer. This can be seen in later times in figure 5.6. The development of this mode is perhaps not surprising considering that the density gradient of the weaker layer remains sharp while an appreceable amount of shear layer vorticity remains. However, it is possible that the growth of the weaker mode has been compromised somewhat due to the periodic streamwise boundary condition. This boundary condition forces the mode to develop at only those wavenumbers that are harmonics of the streamwise domain length $L_{x}$. In general, the results of linear stability analysis show that the weaker mode has maximum growth rates at higher wavenumbers than the dominant mode (Haigh, 1995). The simulation was not carried out for a long enough duration to quantify the behavior of the weaker mode.

The growth of both primary and secondary instabilities can be seen in the $K_{2 d}$ and $K_{3 d}$ curves in figure $5.5 \mathrm{~d}$, respectively. Here the kinetic energy associated with the primary instability, $K_{2 d}$, grows at a near constant exponential rate until $t \approx 25$, corresponding to the time at which the initial billow structure begins to thin. This time marks the beginning of secondary growth given by $K_{3 d}$. The initial growth rate of secondary instabilities can be inferred from the slope of this curve, and is seen to be larger than that of the primary instability. This is in agreement with the results for symmetric stratified shear layers in SW03 and Caulfield and Peltier (2000). As the $K_{3 d}$ reservoir reaches appreciable levels $K_{2 d}$ begins to decay as transition occurs. By the end of the simulation the signature of the weaker mode can be seen in the leveling off of the $K_{2 d}$ curve with a pronounced oscillation. 

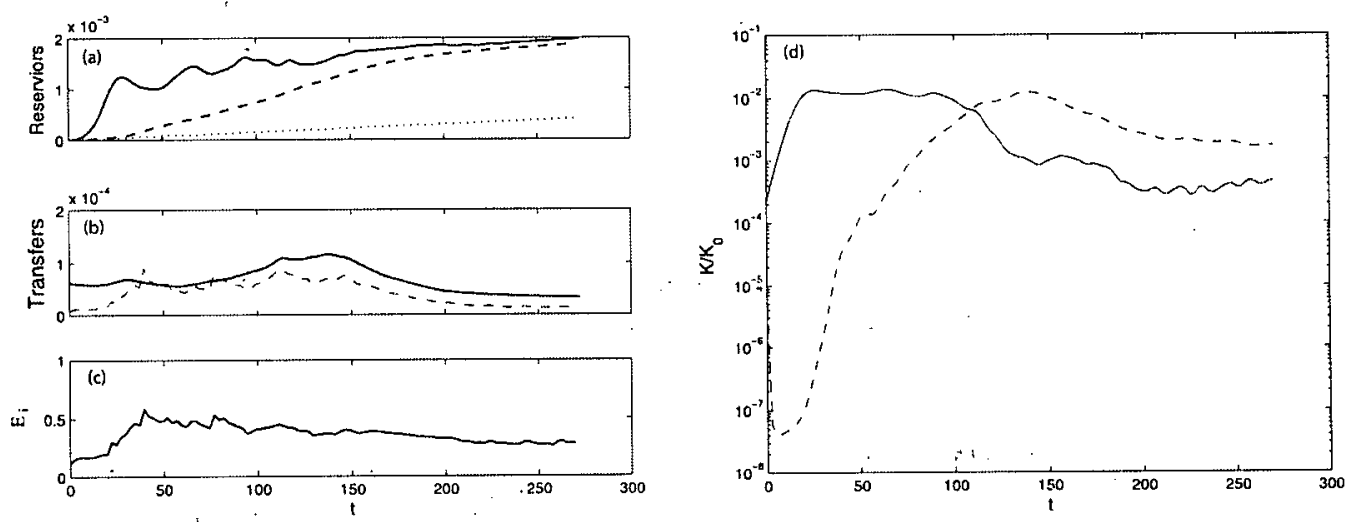

Figure 5.8: Energy reservoirs and transfers for the $\epsilon=0.50$ simulation. The plot in (a) includes the potential energy reservoirs $P$ (solid line), $P_{B}$ (dashed line), and $\Phi$ (dotted line). In (b) both irreversible transfers $D$ (solid line), and $M$ (dashed line) are shown along with $E_{i}$ in (c). The $K_{2 d}$ (solid line) and $K_{3 d}$ (dashed line) are plotted in (d).

$\epsilon=0.50$ case:

As the asymmetry is increased to $\epsilon=0.50$, both similarities and departures from the $\epsilon=0.25$ case can be seen. In figure 5.8 a sharp rise in $P_{A}$ can be seen as a billow structure develops. The quicker formation of the intial instability is consistent with the high growth rate predicted by linear theory. By examining the density field in figure 5.9 , it can be seen that the billow grows to a larger diameter than in the $\epsilon=0.25$ case. This is not surprising since the dominant layer has a greater amount of shear layer vorticity available. The rapid development of the AH instability leads to a considerably larger $P_{A}$ increase in this case, and appears to have overshot the amount of dense fluid that it can entrain in the primary vortex. This is suggested by the thinning of the billow to produce a cusp structure as a portion of the dense entrained fluid sinks to the level of the interface. In this respect the 


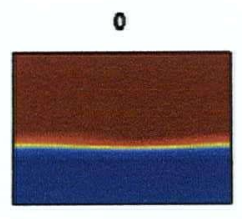

57

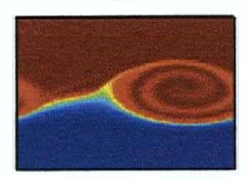

116

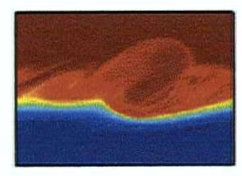

171

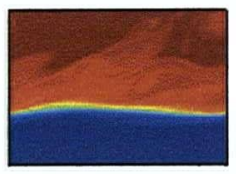

15

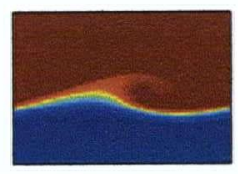

74

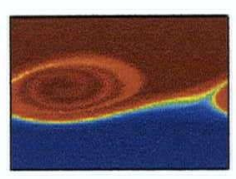

129

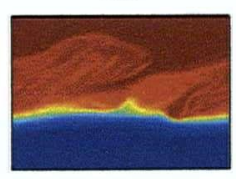

186

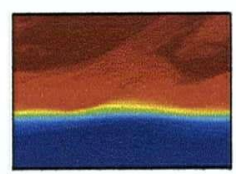

30

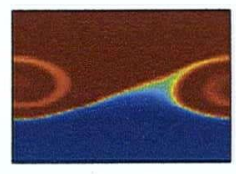

87

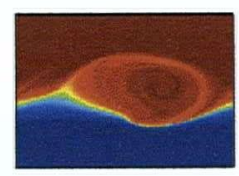

144

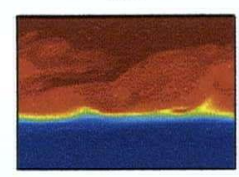

198

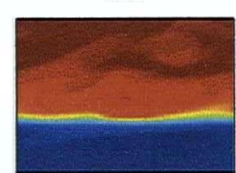

45

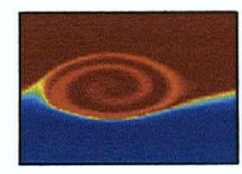

101

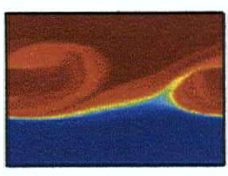

156

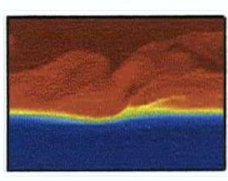

208

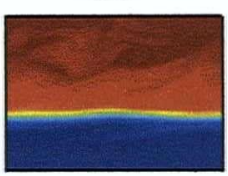

Figure 5.9: Plots of the density field in the $\epsilon=0.50$ simulation showing its time evolution. Slices of the $x z$-plane are taken at $y=L_{y} / 2$ with times shown in each plot. 
initial development of the $\mathrm{AH}$ instability resembles a transient ejection event rather than the steady roll-up of a KH-like billow. This description of the initial development becomes clearer as the asymmetry is increased further, discussed in following paragraphs.

Following initial development, the flow enters a preturbulent period in which the majority of the mixing is accomplished. Just as in the $\epsilon=0.25$ case, this period sees the growth of secondary instabillities leading to the turbulent transition indicated by elevated levels of $D$ starting at $t \approx 100$. Throughout both the preturbulent and turbulent phases the mixing stays relatively constant with small changes present in the turbulent phase and in periods associated with ejection events. Again, the preturbulent phase is found to be the most efficient, with a gradual decline following the development of a more complex flow structure. During the preturbulent phase high levels of mixing are maintained by the 'scraping' of the density interface and subsequent entrainment of this fluid by the primary vortex. Since the entrained fluid is of only intermediate density, there appears to be no saturation of the primary vortex as is found in $\mathrm{KH}$ instabilities. This scraping of the interface leads to sharper scalar gradients and higher mass fluxes.

The amplification of streamwise vorticity by locally unstable regions still appears to be a prominant cause of three-dimensional motions. However, a more complex interaction between the streamwise vortices and the density interface is observed for $\epsilon=0.50$. This consists of a three-dimensional entrainment of fluid from the density interface within the primary vortex as shown in figure 5.10. It is possible that the higher levels of mixing and earlier transition to turbulence that occurs for $\epsilon=0.50$ is a result of this more pronounced entrainment mechanism on the density 
89

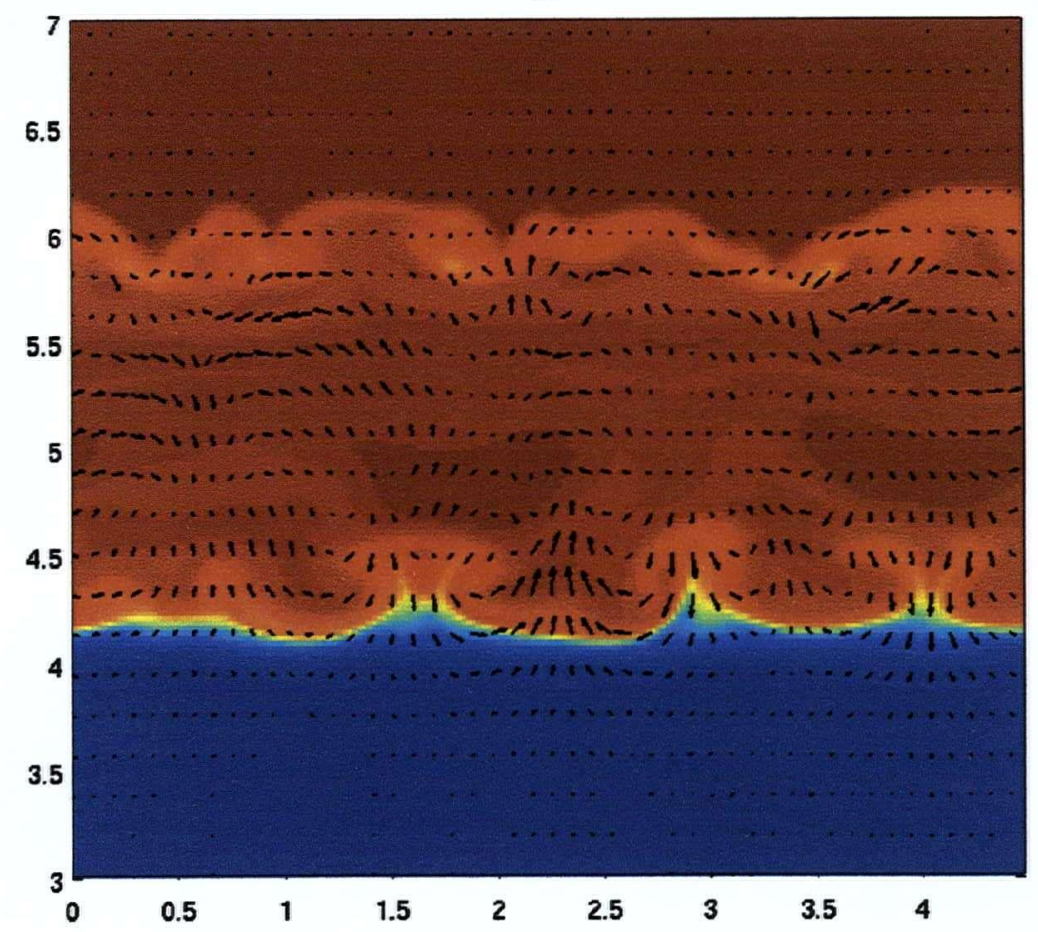

Figure 5.10: Cross-section in the $y z$-plane of the density field with $\vec{u}_{3 d}$ vectors overlain for the $\epsilon=0.50$ simulation. The streamwise location is taken within the primary vortex for $x=4 h$ at $t=89$. 
interface. By examining the growth of $K_{3 d}$ in figure $5.8 \mathrm{~d}$ it can be seen that these two processes are intimately linked. The evolution of $K_{2 d}$ and $K_{3 d}$ appear qualitatively quite similar to the $\epsilon=0.25$ simulation. However, there is a notable change in the growth rate of $K_{3 d}$ beginning at $t \approx 45$ and corresponds to the sinking of the initial ejection to the level of the density interface. In this position the development of three-dimensional motions is suppressed by the stable stratification. The growth of $K_{3 d}$ does not resume until a number of time scales later. This relationship between the growth of three-dimensional motions and ejection events can be seen more clearly as the asymmetry is increased.

$\epsilon=1.0$ case:

A further increase in the asymmetry to $\epsilon=1.0$ results in the energy characteristics shown in figure 5.11. Again, the higher growth rate and larger shear layer vorticity leads to the rapid formation of $P_{A}$. This initial spike drops considerably due to the immediate break-off and vertical settling of the dense fluid at the bottom of the primary vortex as seen in figure 5.12. This supports the view proposed in the previous paragraphs that the initial development of the instability is best though of as an ejection event. It can be characterized by a rise and fall in $P_{A}$ followed by slightly higher local values of $D$ and $M$. (Recall that the transfers $D$ and $M$ are volume averaged quantities so that they are relatively insensitive to spatially localized increases.) This same signature can also be seen at a later time of $t \approx$ $55-75$ and indeed, is visible in the density field of figure 5.12.

As the magnitude of the ejection events increase with increasing asymmetry (and a greater amount of available shear layer vorticity), so too does the transient devel- 

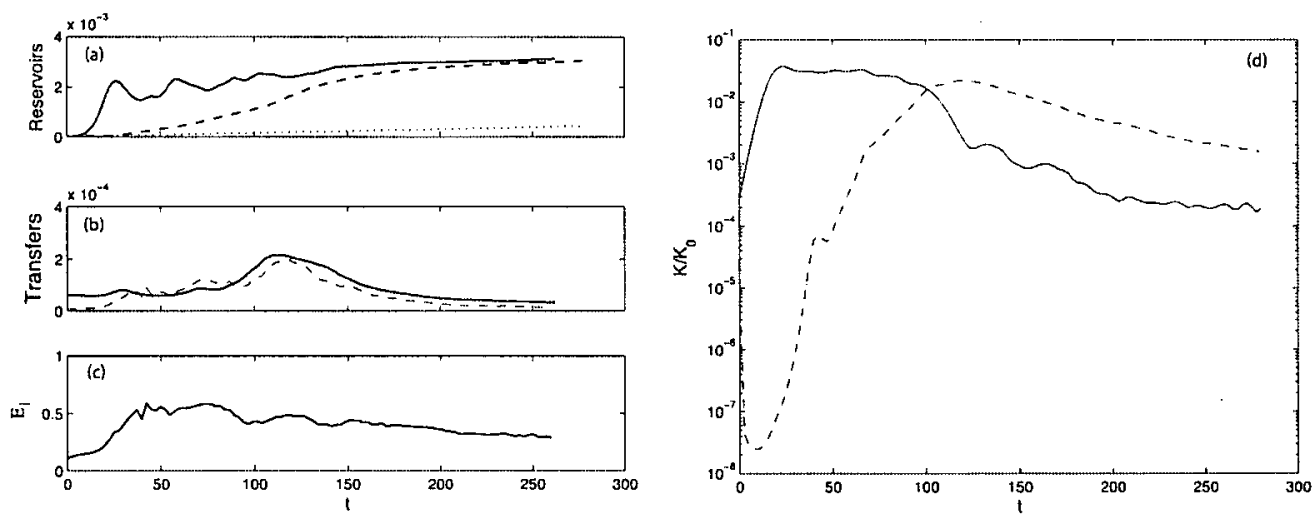

Figure 5.11: Energy reservoirs and transfers for the $\mathrm{KH}$ simulation. The plot in (a) includes the potential energy reservoirs $P$ (solid line), $P_{B}$ (dashed line), and $\Phi$ (dotted line). In (b) both irreversible transfers $D$ (solid line), and $M$ (dashed line) are shown along with $E_{i}$ in (c). The $K_{2 d}$ (solid line) and $K_{3 d}$ (dashed line) are plotted in $(\mathrm{d})$.

opment of three-dimensional motion. The $K_{3 d}$ curve in figure 5.11 shows changes in growth rate corresponding to periods where the ejection has contacted the density interface (i.e., at $t \approx 45$ and $t \approx 65$ ). This reveals support for the damping of three-dimensional motions as the density interface is approached.

\section{$\epsilon>1$ cases:}

Thus far, as the asymmetry is increased so too are the gains in $P_{B}$, as the instabilities extract greater energy from the shear layer they are able to produce greater, more energetic mixing. Intuitively, this relationship cannot hold as the asymmetry is increased indefinitely, since the flow would approach the configuration of a homogenous free shear layer overlying an undisturbed density interface. Also, the pairing of adjacent vortices that is present in homogenous shear layers will also be expected to play a role as $\epsilon$ increases. For these reasons the $\epsilon>1$ simulations serve 

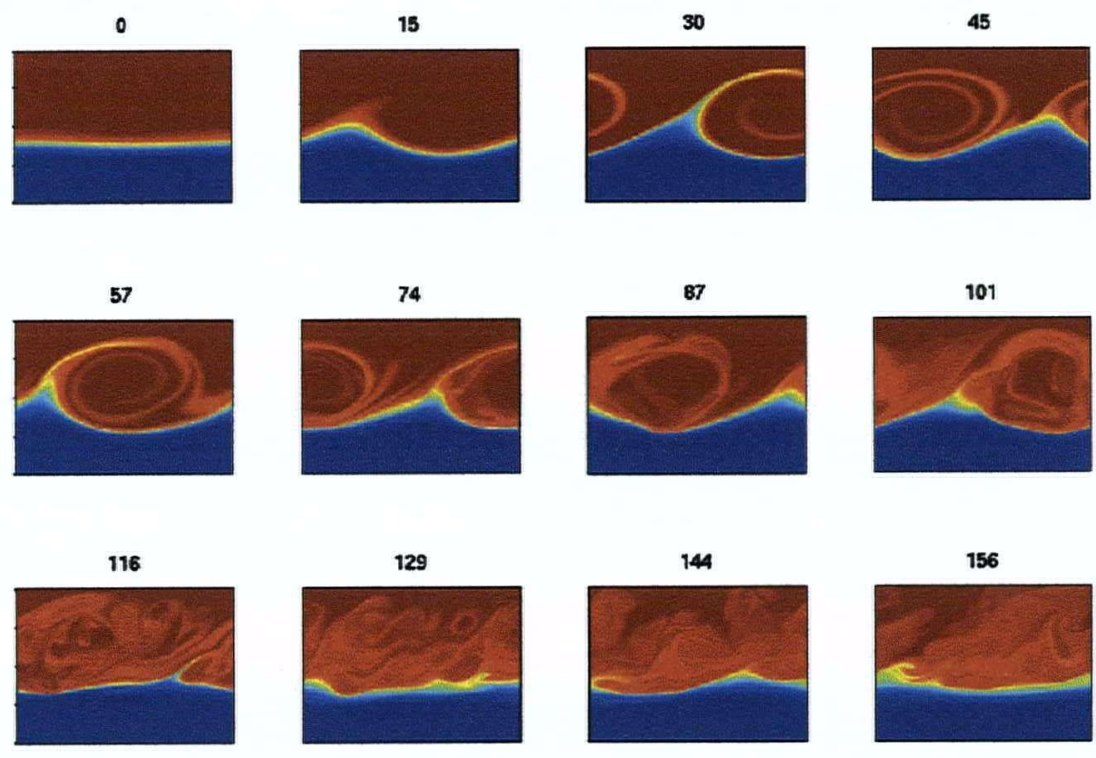

171
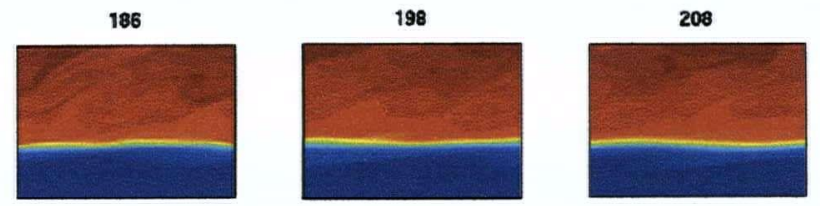

Figure 5.12: Plots of the density field in the $\epsilon=1.0$ simulation showing its time evolution. Slices of the $x z$-plane are taken at $y=L_{y} / 2$ with times shown in each plot. 
only to add a level of completeness to the examination of asymmetry effects. 


\section{Chapter 6}

\section{Discussion}

Results of the numerical simulations enable a direct comparison of the evolution of symmetric and asymmetric instabilities. In particular, a more general description of the mixing behavior is now made. The applicability of the results to geophysical flows is then discussed.

\subsection{Mixing Behavior}

It has been found that mixing by shear instabilities in this region of parameter space can be broken down into two fundamentally different processes that are characteristic of $\mathrm{KH}$ and Holmboe-like modes. In the $\mathrm{KH}$ case, where the thickness of the density interface is of the same order as the thickness of the shear layer (i.e., $R=1$ ), growth of the instability is characterized by significant regions of overturning. It is found to have a short, but efficient, preturbulent phase in which the highest rates of mixing are observed. In this state, the saturated $\mathrm{KH}$ billow has developed secondary instabilities of large amplitude which supply further energy to the mixing process. This phase ends with the turbulent collapse of the localized unstable regions within the billow core. In this sense the instability is self-limiting since the preturbulent phase is relatively short-lived, with greatest mixing occurring just prior to the turbulent collapse. 
In the study by SW03 it was found that the lower primary growth rate of the Holmboe instability led to a longer preturbulent phase where greater mixing was accomplished. Here, a longer preturbulent phase of high efficiency mixing was found to occur even in the $\mathrm{AH}$ instabilities exhibiting larger growth rates. The instability can support more energetic three-dimensional motions before the turbulent transition, leading to greater mixing. This is linked to the entrainment processes identified in $\mathrm{AH}$ instabilities, namely the ejection of intermediate density fluid into the primary vortex, and the 'scraping' of the density interface in the region upstream of the cusp. Both these processes lead to a sharpening of the interface which enhances mixing and possibly helps to maintain the preturbulent form of the instability.

To compare the growth of three-dimensional motions in each simulation figure 6.1 shows the evolution of the $K_{3 d}$ reservoir in each case. It should be noted that the initial growth rates of the $\mathrm{KH}$ and $\mathrm{AH}$ cases are quite similar; an indication that they all result from the generation of statically unstable regions. The $K_{3 d}$ growth of the Holmboe simulation appears to be the result of a different process. It is not clear at this time what mechanism is responsible for the growth of secondary instabilities in the Holmboe case..(see SW03 for a discussion). The ability of AH instabilities to support more energetic three-dimensional motions is indicated by the higher levels of $K_{3 d}$ attained in figure 6.1 .

Though the properties discussed above apply to all AH instabilities in this study to varying degrees, there exist large differences in the total amount of mixing between the various asymmetric cases. The net amount of mixing - measured as the total background potential energy gain due to fluid motions - is plotted for each simulation in figure 6.2. The general trend is the larger the degree of asymme- 


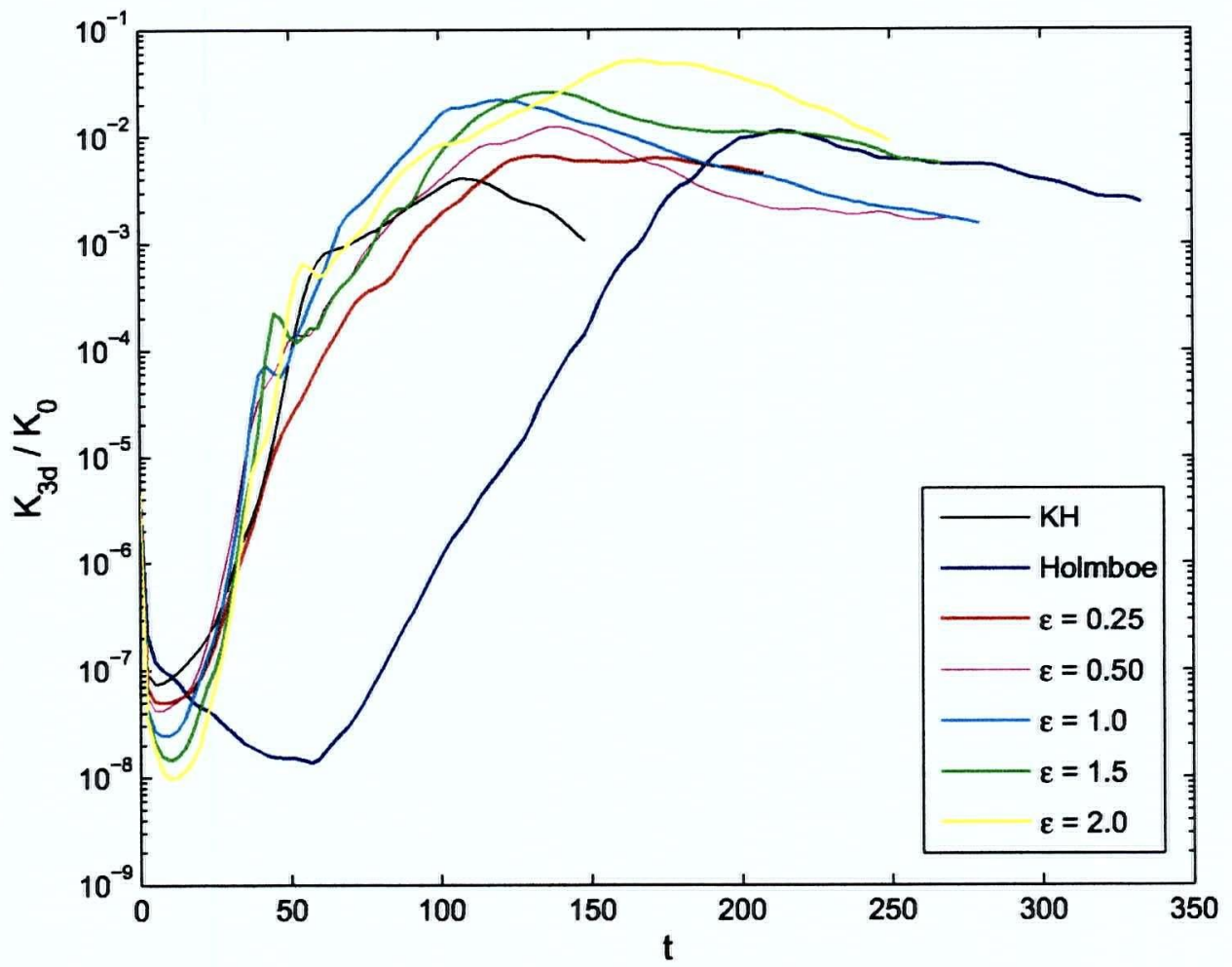

Figure 6.1: Evolution of the $K_{3 d}$ reservoir for each simulation. 


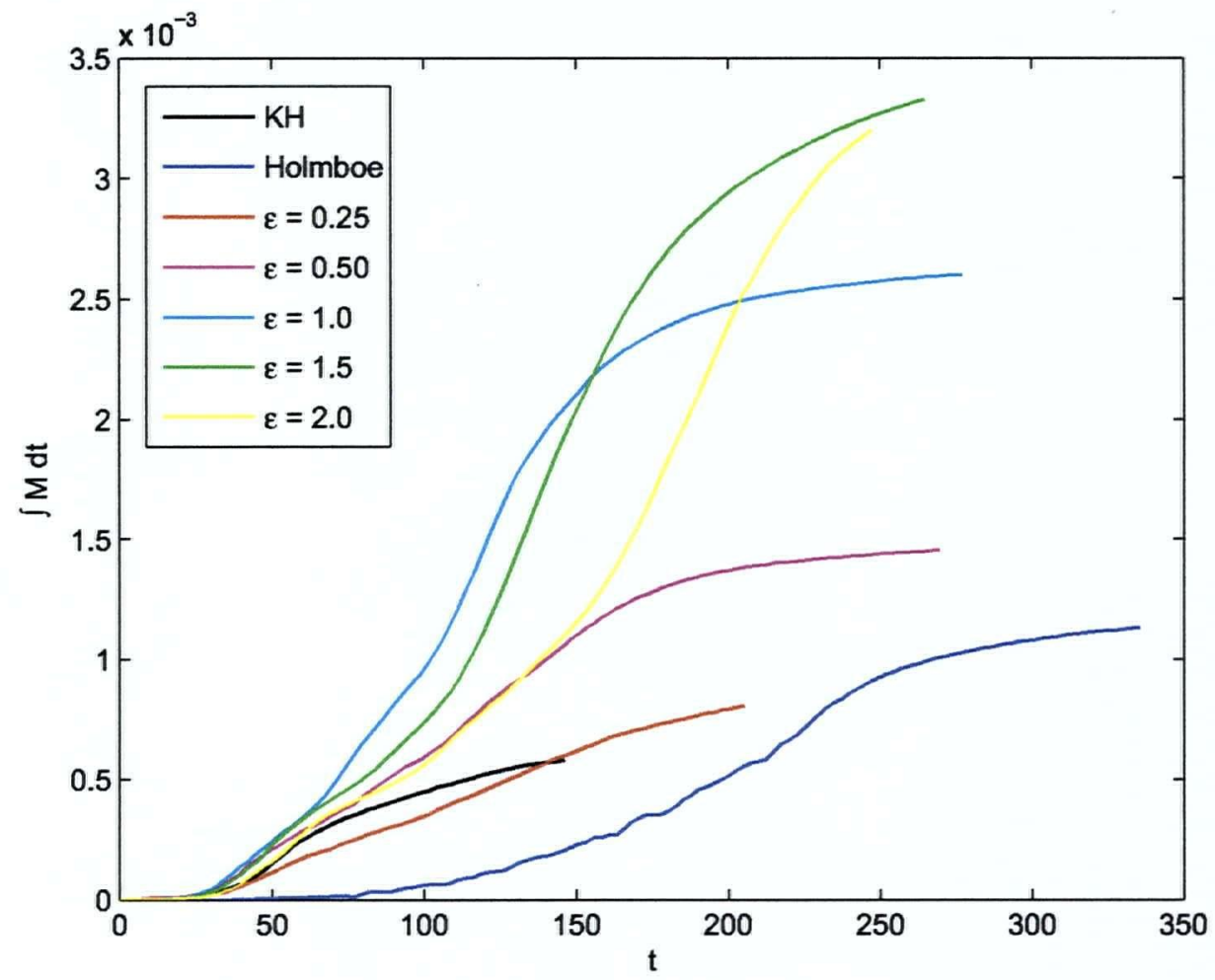

Figure 6.2: Comparison of the total amount of mixing in each simulation. 
try becomes, the more the development of the instability is dominated by shear layer vorticity. In this respect, the larger asymmetries are able to extract a greater amount of energy from the initial shear layer. This can be seen by looking at the total average kinetic energy reservoir, $K$, in each of the asymmetric cases shown in figure 6.3. Since the $K$ reservoir is the energy source of the instability it gives a good

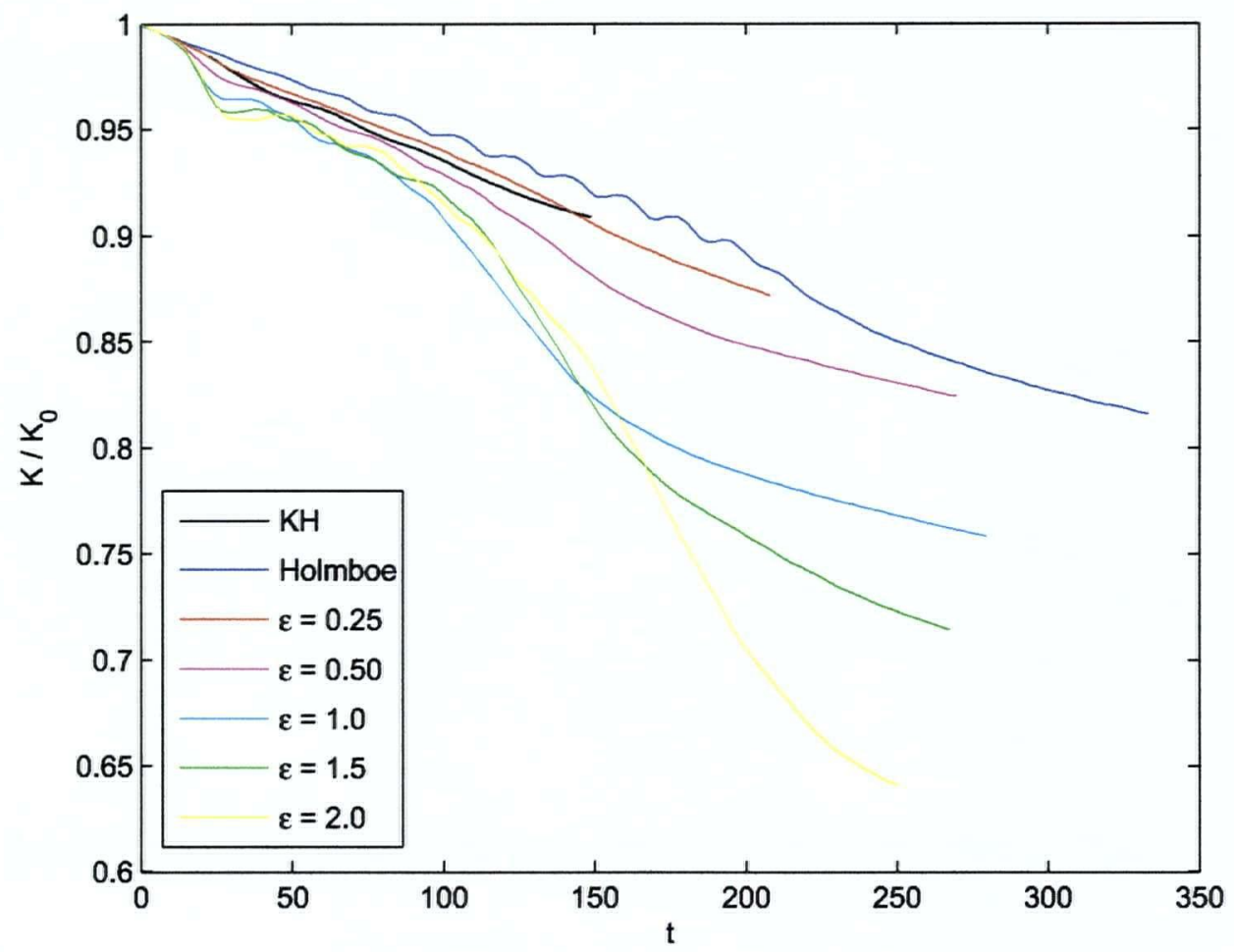

Figure 6.3: Evolution of $K$ as a fraction of the initial kinetic energy $K_{0}=K(0)$ for each simulation.

indication of the levels of extraction in each simulation. These plots show a greater extraction of energy as the asymmetry is increased. This result also agrees with the observations of increasing initial billow diameters and an increased presence of 
ejection events in the cases of greater asymmetry.

Although an increased extraction of energy from the shear layer is observed in cases of greater asymmetry, this does not necessarily lead to a greater mixing of the density field. The proportion of extracted energy that is used to perform mixing is given by the cumulative mixing efficiency, $E_{c}$. Figure 6.4 shows both the net energy gain due to mixing and $E_{c}$ for various asymmetries. Here, increases in $\epsilon$ initially lead
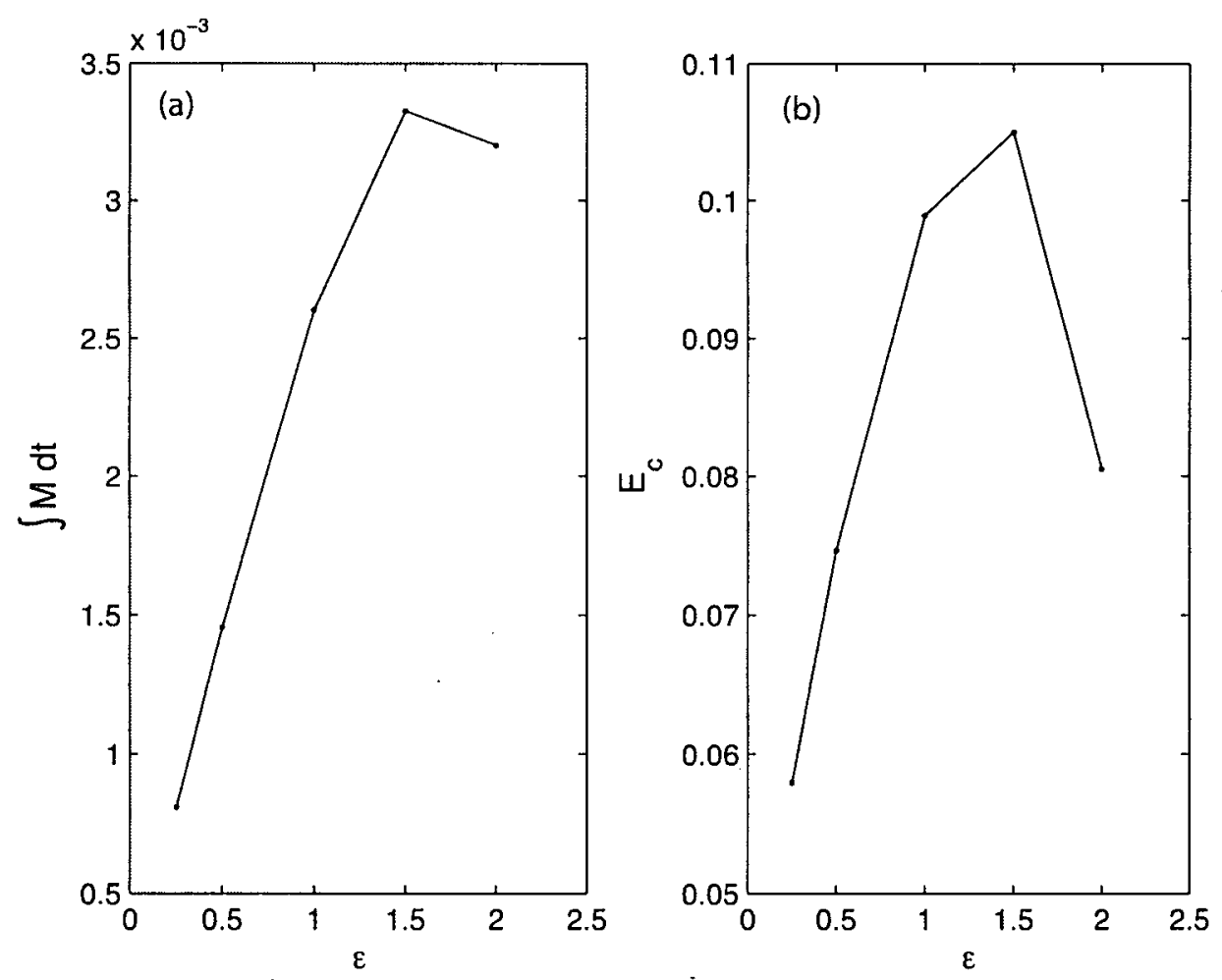

Figure 6.4: Changes in (a) the total mixing and (b) $E_{c}$ for the various asymmetries.

to a more efficient mixing process. This efficiency begins to drop off for $\epsilon \gtrsim 1.5$ and is most likely due to the greater distance between the centre of instability and the density interface. Eventually a point is reached at $\epsilon \approx 2$ where although the highest 
levels of energy are extracted from the shear layer, less mixing is accomplished.

The intuitive notion espoused in SW03 - that for sufficiently large $J$ and $R$, the density interface effectively acts as a flexible barrier to the vorticity of the shear layer - can be seen to support these results. Since this barrier is sufficiently strong, it prevents communication between layers, and isolates the vorticity in the weaker layer. By this process the extraction of energy in the $\mathrm{AH}$ instability is limited to the shear of the dominant layer until the formation of the weaker mode. As the asymmetry is increased past $\epsilon \gtrsim 1.5$ the growth of the instability is increasingly isolated from the density interface and cannot perform mixing as efficiently. If $\epsilon$ were to continue to increase, the instability would approach that of an homogenous shear layer with a vanishing mixing efficiency.

In accordance with the theoretical and laboratory investigation of Lawrence et al. (1991) the development of AH instabilities resulted in a distinct one-sidedness. The final result of this one-sidedness is a greater mixing of the density profile in the dominant layer. This can be seen in the final averaged density profiles shown in figure 6.5 where the initial profile is included for comparison. The vertical distribution of density elements throughout the computational domain is also shown in figure 6.5 for both the initial and final simulation times. These plots indicate that the mixing processes in $\mathrm{AH}$ instabilities are responsible for the production of largely low-density fluid (since the dominant layer coincides with the low-density stream in this case). This confirms the observation that it is fluid from the upper portions of the interface that is mixed in the upper layer. This process results in a slight deepening of the upper layer in which the effects can be noticed at a distance of $\approx 2.5-3.5$ shear layer depths from the new interface position. The weaker layer is left relatively 
(a)

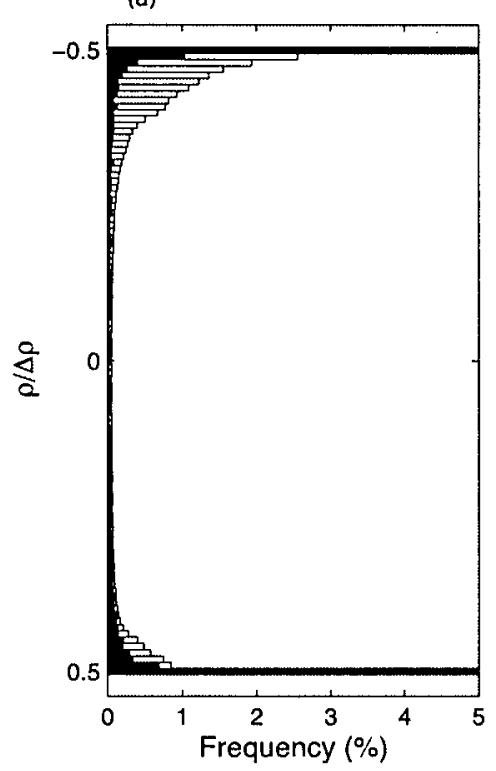

(c)

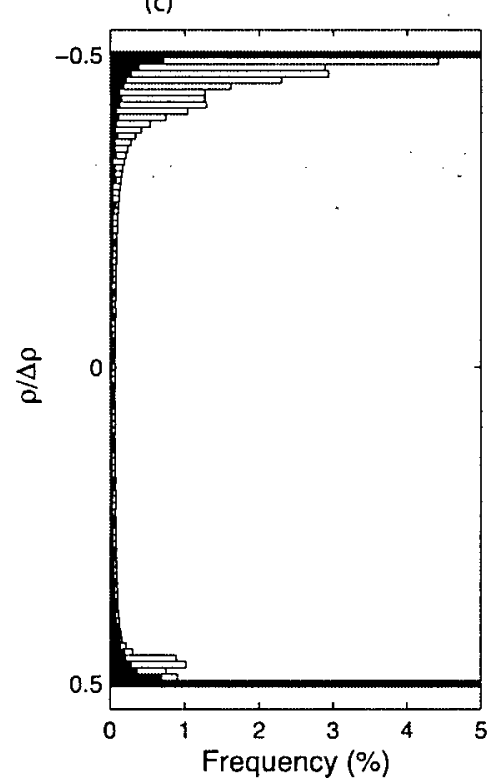

(b)

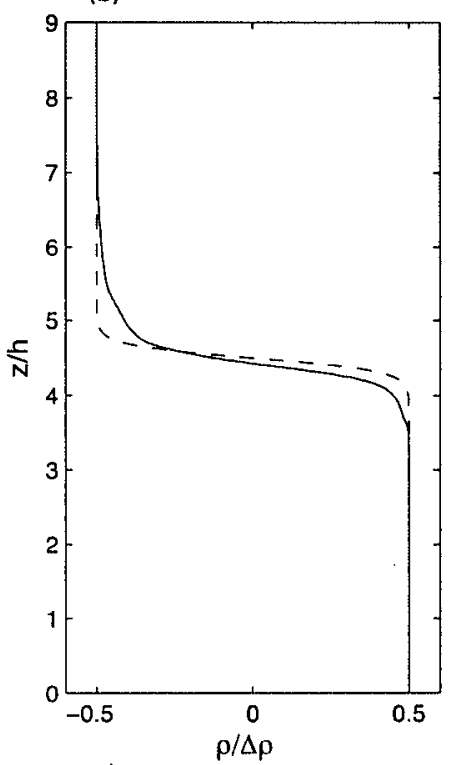

(d)

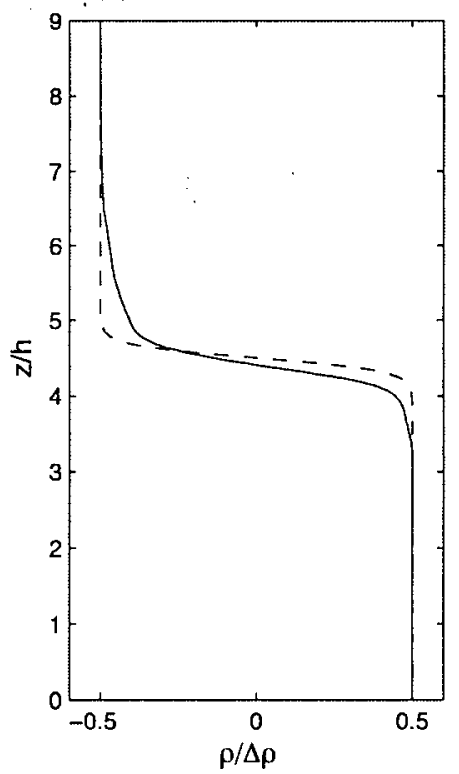

Figure 6.5: Initial and final density characteristics of the asymmetric simulations for $\epsilon=0.25$ in (a) and (b), $\epsilon=0.50$ in (c) and (d). In (a) and (c) the distribution of density elements is shown for the initial time (dark bars) and the final time (light bars). In (b) and (d) the initial (dashed line) and final (solid line) density profiles are given. All density values are given as the difference from the initial mean density. 
untouched with a sharp gradient still intact. This should allow for the formation of the weaker mode sometime after the dominant mode has stabilized, as was observed in the $\epsilon=0.25$ and $\epsilon=0.50$ cases.

The different mixing behavior of the $\mathrm{KH}$ instability can also be seen to manifest itself in the final density distribution, shown in figure 6.6a and 6.6b. Here the mixing process is responsible for producing exclusively intermediate density fluid. This observation was noted in $\S 5.1$ as the mixing was concentrated primarily within the billow core and originated from the high- and low-density fluid of the edges of the interface and shear layer. The increase in intermediate density fluid leaves a steplike structure in the profile with two locations of higher gradients. See Caulfield and Peltier (2000) for a discussion of this density profile generation in $\mathrm{KH}$ instabilities. It should also be noted that the vertical extent of the mixing is entirely within the original shear layer and density interface thickness - in marked contrast to the $\mathrm{AH}$ cases. The Holmboe simulation (figures $6.6 \mathrm{c}$ and $6.6 \mathrm{~d}$ ) displays mixing behavior similar to the $\mathrm{AH}$ cases in that the final density distribution shows mixing concentrated above and below the interface.

The difference between mixing behavior in $\mathrm{AH}$ and Holmboe instabilities and that of KH instabilities was first noticed by Thorpe (1968). Here it was observed that in certain mixing events, the density interface 'retains its identity', meaning that mixing is accomplished without the collapse of overturned regions. In this case there is no mixing of fluid across the interface - as is the dominant process in $\mathrm{KH}$ instabilities. 
(a)

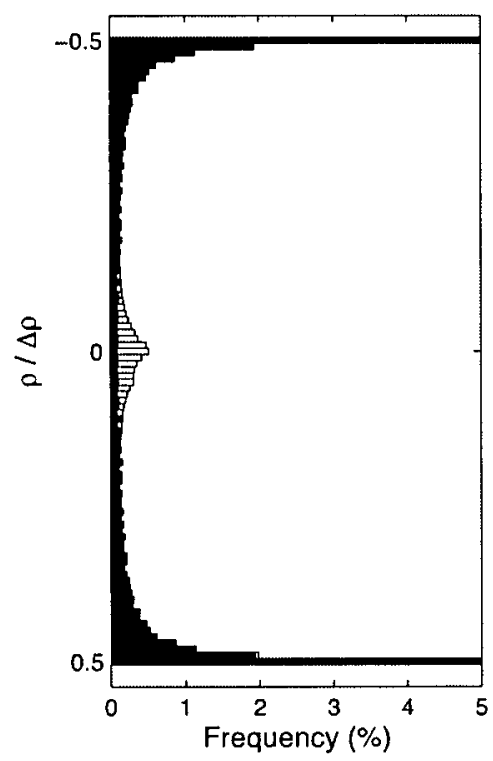

(c)

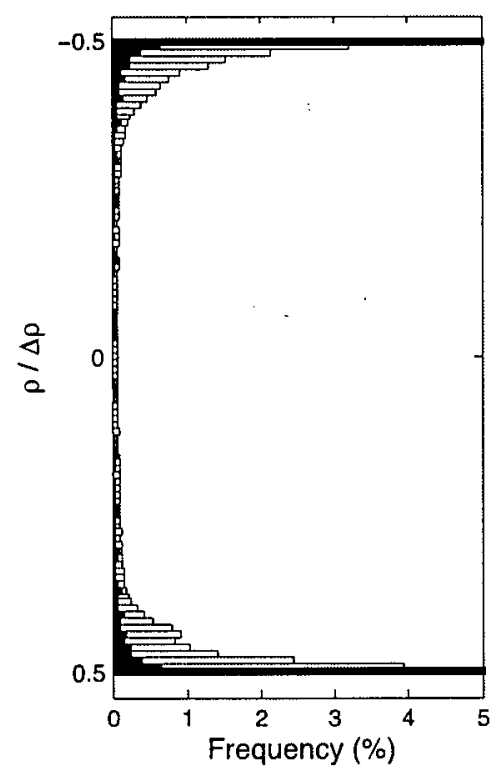

(b)

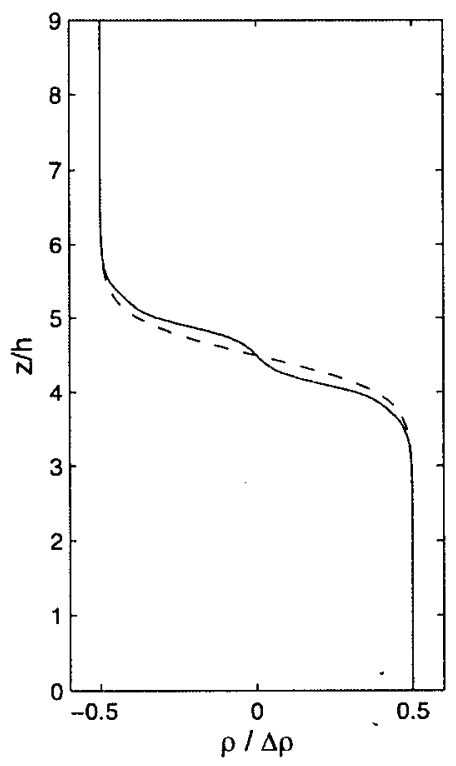

(d)

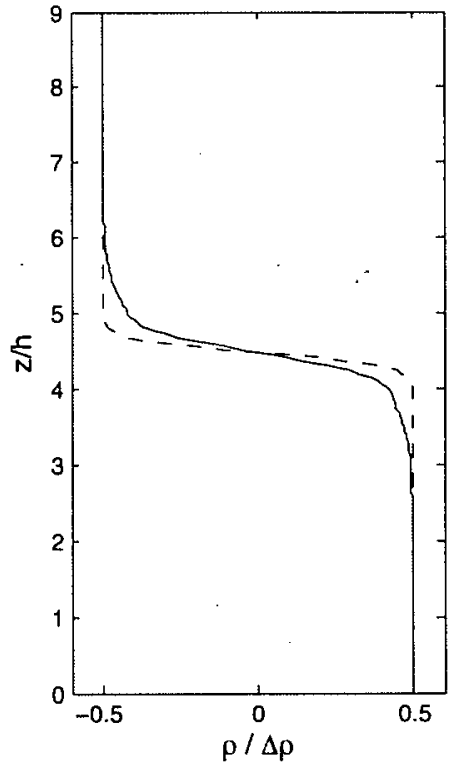

Figure 6.6: Initial and final density characteristics of the symmetric simulations. In (a) and (c) the distribution of density elements is shown for the initial time (dark bars) and the final time (light bars). The initial (dashed line) and final (solid line) density profiles are given in (b) and (d). The KH simulation corresponds to (a) and (b), whereas the Holmboe is depicted in (c) and (d). All density values are given as the difference from the initial mean density. 


\subsection{Relevance to Geophysical Flows}

The objective of the present study is to examine the mixing and turbulent transition in asymmetric shear layers. This is motivated in part by the occurance of asymmetry in flows of significant geophysical importance, such as exchange flows and salt-wedge intrusions, as discussed in $§ 3$. However, numerical limitations due to the present state of computational technology (see $\S 4.1$ ) requires the simulations to be run at lower $R e$ and $\operatorname{Pr}$ than would normally be expected in natural settings. Although the values used in this study are representative of low $R e$ events that are within (but at the low end of) the range observed in the oceanic thermocline by Smyth et al. (2001), and in the seasonal Mediterranean thermocline by Woods (1968) (see also Thorpe (1985)).

Questions concerning the applicability of DNS in shear layers for $R e<10^{4}$ have been voiced in Moin and Mahesh (1998). This concerns a phenomenon that has been termed the 'mixing transition'. It describes what is thought to a be a universal attribute of turbulent flows whereby once a certain $R e \sim O\left(10^{4}\right)$ is exceeded, "a qualitative difference in the appearance of the scalar field is observed ... which results in a more well-mixed state" (Dimotakis, 2000).

The mixing transition has been well documented in homogenous free shear layers (see Dimotakis (2000) and references therein) but its extension to stratified shear layers remains to be fully quantified. The numerical simulations of Peltier and Caulfield (2003) examine the development of KH billows for a range of $J$. In these simulations a qualitative change in the behavior of the flow is noted for the low- $J$ simulations as three-dimensionality is achieved. Since $R e \sim O\left(10^{4}\right)$ for these flows, effects of the mixing transition can be expected (Peltier and Caulfield, 2003). 
The onset of three-dimensional motion coincident with a qualitative change in the behavior of the flow has been noted previously by Breidenthal (1981). Subsequent to this transition, a period of intense turbulence is observed in which the majority of the mixing is achieved. However, in the simulations of Peltier and Caulfield (2003) as the stratification is increased such that $J>0.1$ the growth of turbulent kinetic energy becomes inhibited by buoyancy forces and the mixing in the turbulent phase becomes less significant. This result certainly agrees with the behavior observed in the asymmetric mixing studied herein. Therefore, the observed importance of the preturbulent phase consisting of a coherent mixing behavior, although influenced by the low $R e$, is not necessarily expected to be a result of the low Re. Further study of the role that stratification plays in the mixing transition is required to assess the geophysical significance of low-Re mixing. 


\section{Chapter 7}

\section{Conclusions and Future Work}

A number of direct numerical simulations have been performed in which the effects of asymmetry on the evolution and mixing behavior of stratified shear instabilities has been examined. The results are compared and contrasted to the two symmetric instabilities that occur at this point in parameter space, namely, the KH and Holmboe instabilities. Two different mixing mechanisms are found to emerge. The first is present only in the $\mathrm{KH}$ case, and consists of the formation of significant regions of overturning. Mixing generated in the collapse of this overturn results in the production of intermediate density fluid, and a layered density profile. The second mixing mechanism is found in $\mathrm{AH}$ instabilities. It is characterized by a sharpening of the density interface by a spanwise vortex, and the entrainment of partially mixed fluid from the edge of the interface. The later of these processes is accomplished largely by periodic ejection events. Both mechanisms were found to have the highest rates of mixing in the preturbulent phase, while the density structure was still coherent. High levels of mixing observed in the AH instabilities are the result of a long lasting preturbulent phase similar to the symmetric Holmboe instability.

As the level of asymmetry is increased more shear layer vorticity becomes involved in the development of the dominant $\mathrm{AH}$ mode. This results in a greater initial extraction of energy from the shear layer producing higher levels of both mixing and viscous dissipation. In cases exhibiting lower asymmetries the weaker 
$\mathrm{AH}$ mode is found to develop after the turbulent breakdown of the dominant mode. This is only possible due to the one-sided mixing behavior of the dominant $\mathrm{AH}$ mode which leaves the density interface sharp in the weaker layer. As first pointed out by Thorpe (1968), the density interface is able to "retain its identity" in the AH mixing process as a result of this one-sided mixing behavior. The evolution of the flow and additional mixing associated with the weaker mode has not been studied in detail.

Three-dimensional structure in the dominant $\mathrm{AH}$ modes was found to consist of streamwise vortices generated by the locally unstable density regions of the $\mathrm{AH}$ billow structure. These structures appear to be influenced by the periodic ejections of fluid from the cusp region and are similar to those observed in the laboratory experiments of Schowalter et al. (1994). The interaction of the streamwise vortices with the density interface - particularly during the sinking of ejections to the interface level - was found to influence the growth of the kinetic energy of the secondary structures. These processes have further implications for the turbulent transition in AH instabilities that have not been fully quantified.

Consideration of the features mentioned above have led to the conclusion that the degree of asymmetry present in the velocity and density profiles is an important factor in the mixing behavior and turbulent transition in stratified shear flows. However, the study of asymmetry in these flows is far from complete. The present study has focused entirely on a single point in parameter space. A more thorough examination would include a number of points throughout the bulk Richardson number domain as well as exploring Reynolds number effects. This inevitably leads to a consideration of the mixing transition in stratified shear layers and is hampered 
numerically by the difficulties in simulating high- $R e$ flows.

While future work may look to expanding the domain of study there are still many unanswered questions regarding the present work. This includes consideration of the pairing mechanism in $\mathrm{AH}$ instabilities. If, as $\epsilon$ is increased, the flow approaches that of a homogenous shear layer where pairing is known to occur, then at what value of $\epsilon$ does pairing become important for asymmetric flows? And what dependence does this value have on the Richardson number and other parameters?

Another area that requires further investigation is the formation of three-dimensional secondary structures and their role in the turbulent transition. Although many similarities have been observed to the existing work on streamwise vortices (see Caulfield and Peltier (2000), Peltier and Caulfield (2003), Schowalter et al. (1994)), the interaction of these structures with the density interface and periodic ejection events remain to be quantified.

Since this study was carried out with the intent of shedding light on mixing processes that occur in flows of a geophysical nature, it is hoped that further research is done to examine $\mathrm{AH}$ instabilities as they are found in nature. It is expected that this research serve as a point of comparison for both numerical and laboratory studies of $\mathrm{AH}$ instabilities as well as providing additional insight into their geophysical significance. 


\section{Bibliography}

Baines, P., and H. Mitsudera, On the mechanism of shear flow instabilities, Journal of Fluid Mechanics, 276, 327-342, 1994.

Batchelor, G., Small-scale variation of convected quantities like temperature in turbulent fluid, Journal of Fluid Mechanics, 5, 113-133, 1959.

Bernal, L., and A. Roshko, Streamwise vortex structures in plane mixing layers, Journal of Fluid Mechanics, 170, 499-525, 1986.

Breidenthal, R., Structure in turbulent mixing layers and wakes using a chemical reaction, Journal of Fluid Mechanics, 109, 1-24, 1981.

Caulfield, C., and W. Peltier, The anatomy of the mixing transition in homogenous and stratified free shear layers, Journal of Fluid Mechanics, 413, 1-47, 2000.

Dimotakis, P., The mixing transition in turbulent flows, Journal of Fluid Mechanics, 409, 69-98, 2000.

Drazin, P., and W. Reid, Hydrodynamic Stability, first paperback ed., Cambridge University Press, 1982.

Farmer, D., and L. Armi, The flow of atlantic water through the strait of gibraltar, Progress in Oceanography, 21, 1-98, 1998. 
Goldstein, S., On the stability of superposed streams of fluids of different densities, Proceedings of the Royal Society of London A, 132, 524-548, 1931.

Haigh, S., Non-symmetric holmboe waves, Ph.D. thesis, University of British Columbia, 1995.

Haigh, S., and G. Lawrence, Symmetric and nonsymmetric holmboe instabilities in an inviscid flow, Physics of Fluids, 11, 1459-1468, 1999.

Helmholtz, H. v., On discontinuous movements of fluids, The London, Edinburgh, and Dublin Philisophical Magazine and Journal of Science, 36, 337-346, 1868.

Hogg, A. M., and G. Ivey, The kelvin-helmholtz to holmboe instability transition in stratified exchange flows, Journal of Fluid Mechanics, 477, 339-362, 2003.

Holmboe, J, On the behavior of symmetric waves in stratified shear layers, Geofys. Publ., pp. 67-112, 1962.

Howard, L., and S. Maslowe, Stability of stratified shear flows, Boundary-Layer Meteorology, 4, 511-523, 1973.

Kelvin, W., Hydrokinetic solutions and observations, The London, Edinburgh, and Dublin Philisophical Magazine and Journal of Science, 42, 362-377, 1871.

Koochesfahani, M. M., and P. Dimotakis, Mixing and chemical reactions in a turbulent liquid mixing layer, Journal of Fluid Mechanics, 170, 83-112, 1986.

Lawrence, G., F. Browand, and L. Redekopp, The stability of a sheared density interface, Physics of Fluids, 3, 2360-2370, 1991. 
Lawrence, G., S. Haigh, and Z. Zhu, In search of holmboe's instability, in Physical Processes in Lakes and Oceans, vol. 54 of Coastal and Estuarine Studies, pp. 295304, American Geophysical Union, 1998.

Maslowe, S., and J. Thompson, Stability of a stratified free shear layer, Physics of Fluids, 14, 453-458, 1971.

Maxworthy, T., and F. Browand, Experiments in rotating and stratified flows: oceanographic application, Annual Review of Fluid Mechanics, 7, 273-305, 1975.

Moin, P., and K. Mahesh, Direct numerical simulation: a tool in turbulence research, Annual Review of Fluid Mechanics, 30, 539-578, 1998.

Pawlak, G., and L. Armi, Vortex dynamics in a spatially accelerating shear layer, Journal of Fluid Mechanics, 376, 1-35, 1998.

Peltier, W., and C. Caulfield, Mixing efficiency in stratified shear flows, Annual Review of Fluid Mechanics, 35, 135-167, 2003.

Rosenhead, L., Formation of vortices from a surface of discontinuity, Proceedings of the Royal Society of London A, 134, 170-192, 1931.

Sargent, F., and G. Jirka, Experiments on saline wedge, Journal of Hydraulic Engineering, 113, 1307-1324, 1987.

Schowalter, D., C. V. Atta, and J. Lasheras, A study of streamwise vortex structure in a stratified shear layer, Journal of Fluid Mechanics, 281, 247-291, 1994.

Smyth, W., and K. Winters, Turbulence and mixing in holmboe waves, Journal of Physical Oceanography, 33, 694-711, 2003. 
Smyth, W., G. Klaassen, and W. Peltier, Finite amplitude holmboe waves, Geophysical and Astrophysical Fluid Dynamics, 43, 181-222, 1988.

Smyth, W., J. Moum, and D. Caldwell, The efficiency of mixing in turbulent patches: Inferences from direct simulations and microstructure observations, Journal of Physical Oceanography, 31, 1969-1992, 2001.

Smyth, W., J. Nash, and J. Moum, Differential diffusion in breaking kelvinhelmholtz billows, Journal of Physical Oceanography, submitted, 2004.

Taylor, G., Effect of variation in density on the stability of superposed streams of fluid, Proceedings of the Royal Society of London A, 132, 499-523, 1931.

Thorpe, S., A method of producing a shear flow in a stratified fluid, Journal of Fluid Mechanics, 32, 693-704, 1968.

Thorpe, S., Laboratory observations of secondary structures in kelvin-helmholtz billows and consequences for ocean mixing, Geophysical and Astrophysical Fluid Dynamics, 34, 175-199, 1985.

Thorpe, S., Transitional phenomena and the development of turbulence in stratified fluids: A review, Journal of Geophysical Research, 92, 5231-5248, 1987.

Turner, J., Buoyancy Effects in Fluids, first paperback ed., Cambridge University Press, 1973.

Winters, K., and H. Seim, The role of dissipation and mixing in exchange flow through a contracting channel, Journal of Fluid Mechanics, 407, 265-290, 2000. 
Winters, K., P. Lombard, J. Riley, and E. D'Asaro, Available potential energy and mixing in density-stratified fluids, Journal of Fluid Mechanics, 289, 115-128, 1995.

Winters, K., J. MacKinnon, and B. Mills, A spectral model for process studies of rotating, density-stratified flows, Journal of Atmospheric and Oceanic Technology, 21, 69-94, 2004.

Woods, J., Wave-induced shear instability in the summer thermocline, Journal of Fluid Mechanics, 32, 791-800, 1968.

Yonemitsu, N., G. Swaters, N. Rajaratnam, and G. Lawrence, Shear instabilities in arrested salt-wedge flows, Dynamics of Atmospheres and Oceans, 24, 173-182, 1996.

Yoshida, S., M. Ohtani, S. Nishida, and P. Linden, Mixing processes in a highly stratified river, in Physical Processes in Lakes and Oceans, vol. 54 of Coastal and Estuarine Studies, pp. 389-400, American Geophysical Union, 1998. 